Homology, Homotopy and Applications, vol.20(1), 2018, pp.303-328

\title{
SMALL PRESENTATIONS OF MODEL CATEGORIES AND VOPĚNKA'S PRINCIPLE
}

\author{
G. RAPTIS AND J. ROSICKÝ
}

(communicated by J. Daniel Christensen)

\begin{abstract}
We prove existence results for small presentations of model categories generalizing a theorem of D. Dugger from combinatorial model categories to more general model categories. Some of these results are shown under the assumption of Vopěnka's principle. Our main theorem applies, in particular, to cofibrantly generated model categories where the domains of the generating cofibrations satisfy a slightly stronger smallness condition. As a consequence, assuming Vopěnka's principle, such a cofibrantly generated model category is Quillen equivalent to a combinatorial model category. Moreover, if there are generating sets which consist of presentable objects, then the same conclusion holds without the assumption of Vopěnka's principle. We also correct a mistake from previous work that made similar claims.
\end{abstract}

\section{Introduction}

The authors addressed in previous articles $[\mathbf{1 6}, \mathbf{1 9}]$ the question of whether a cofibrantly generated model category is Quillen equivalent to a combinatorial model category and connected this question with special set-theoretical assumptions. More specifically, the first author claimed in [16] that assuming Vopěnka's principle any cofibrantly generated model category is Quillen equivalent to a combinatorial one. The proof appeared to generalize also to a weaker notion of a cofibrantly generated model category where the domains of the generating sets need not be small. The second author claimed in [19] that Vopěnka's principle is equivalent to the statement that such a generalized cofibrantly generated model category admits a combinatorial model up to Quillen equivalence. Unfortunately, the proof in [16] contains a mistake which cannot be repaired without adding further assumptions.

The purpose of the present article is to point out this mistake and present a different approach to the problem of finding combinatorial models for cofibrantly generated model categories. Our main theorem establishes a slightly weaker version of the claim in [16] but it applies to more general model categories as well. Moreover, we show that there are examples of cofibrantly generated model categories in the

Received July 11, 2017, revised October 10, 2017; published on February 28, 2018.

2010 Mathematics Subject Classification: 18G55, 18C35, 55U35.

Key words and phrases: cofibrantly generated model category, combinatorial model category, simplicial presheaves, Vopěnka's principle.

Article available at http://dx.doi.org/10.4310/HHA.2018.v20.n1.a18

Copyright (c) 2018, International Press. Permission to copy for private use granted. 
generalized sense of [19], which do not admit a combinatorial model, independently of Vopěnka's principle.

Our main results are based on a delicate analysis of the properties of weak factorization systems that are generated by sets of morphisms and which satisfy various kinds of smallness conditions. We introduce a hierarchy of smallness conditions, as well as associated notions of cofibrantly generated model categories, and investigate their implications for the categories of cofibrant objects and for the classes of weak equivalences. Recall that a weak factorization system $(\mathcal{L}, \mathcal{R})$ in a cocomplete category $\mathcal{M}$ is cofibrantly generated if there is a set of morphisms $\mathcal{X} \subseteq \mathcal{L}$ such that

(a) the domains of $\mathcal{X}$ are small relative to $\mathcal{X}$,

(b) $\mathcal{X}^{\square}=\mathcal{R}$.

This corresponds to the standard notion that appears in the definition of a cofibrantly generated model category. We introduce the following variants of this definition (see Definition 2.9 for a more precise definition and a more complete list):

(1) $(\mathcal{L}, \mathcal{R})$ is weakly cofibrantly generated if there is a set $\mathcal{X} \subseteq \mathcal{L}$ such that $\mathcal{L}=$ $\operatorname{cof}(\mathcal{X})$ where $\operatorname{cof}(\mathcal{X})$ denotes the smallest class of morphisms in $\mathcal{M}$ which contains $\mathcal{X}$ and is closed under pushouts, transfinite compositions, and retracts.

(2) $(\mathcal{L}, \mathcal{R})$ is strictly cofibrantly generated if there is a set $\mathcal{X} \subseteq \mathcal{L}$ that satisfies (b) and the domains of the morphisms in $\mathcal{X}$ are small with respect to $\lambda$-directed good colimits whose links are $\mathcal{X}$-cellular morphisms.

(3) $(\mathcal{L}, \mathcal{R})$ is perfectly cofibrantly generated if there is a set $\mathcal{X} \subseteq \mathcal{L}$ that satisfies (b) and the domains and the codomains of the morphisms in $\mathcal{X}$ are presentable objects in $\mathcal{M}$.

The notion of a good colimit will be recalled in Section 2. The smallness condition in (2) appears to be stronger than the one in (a) above, at least when the corresponding smallness rank is uncountable. Roughly speaking, while the smallness condition in (a) is concerned with directed chains of $\mathcal{X}$-cellular morphisms, the one in (2) applies also to wider directed diagrams of $\mathcal{X}$-cellular morphisms. This seems to be a natural variant of the standard definition from the viewpoint of the fat small object argument [14].

Model categories were first introduced and studied by Quillen in his seminal work [15]. Following the standard conventions from the more recent treatments of the subject $[\mathbf{9}, \mathbf{1 0}]$, a model category consists of a complete and cocomplete category $\mathcal{M}$ together with three classes of morphisms $\mathcal{C}$ of, $\mathcal{W}$ and $\mathcal{F} i b$ such that $\mathcal{W}$ contains the isomorphisms and has the 2-out-of-3 property and the two pairs $(\mathcal{C} \circ f \cap \mathcal{W}, \mathcal{F} i b)$ and $(\mathcal{C} o f, \mathcal{W} \cap \mathcal{F} i b)$ define weak factorization systems with functorial factorizations. A model category $(\mathcal{M}, \mathcal{C} o f, \mathcal{W}, \mathcal{F} i b)$ is cofibrantly generated if both weak factorization systems are cofibrantly generated. Moreover, $(\mathcal{M}, \mathcal{C} \circ f, \mathcal{W}, \mathcal{F} i b)$ is a combinatorial model category if it is cofibrantly generated and its underlying category $\mathcal{M}$ is locally presentable. Examples of combinatorial model categories include the standard model category of simplicial sets $\mathcal{S} \mathcal{S}$ et, the projective model category of simplicial presheaves $\mathcal{S} \mathcal{S} \mathrm{et}^{{ }^{\mathrm{OP}}}$ where $\mathcal{C}$ is a small category, and the left Bousfield localization $L_{S} \mathcal{S} \mathcal{S e t}^{\mathcal{C}^{\text {op }}}$ of $\mathcal{S} \mathcal{S e t}^{\mathcal{C}^{\mathrm{op}}}$ at a set of morphisms $S$.

A small presentation of a model category $(\mathcal{M}, \mathcal{C} o f, \mathcal{W}, \mathcal{F} i b)$ consists of a small 
category $\mathcal{C}$, a set of morphisms $S$ in $\mathcal{S} \mathcal{S}$ et ${ }^{\mathcal{C}^{\text {op }}}$ and a Quillen equivalence

$$
F: L_{S} \mathcal{S} \mathcal{S} \mathrm{et}^{\mathcal{C}^{\mathrm{op}}} \rightleftarrows \mathcal{M}: G \text {. }
$$

This notion was introduced by Dugger [5] who showed that every combinatorial model category admits a small presentation. In this paper, we extend this result to more general model categories and in this way we conclude that these model categories admit combinatorial models. Our main results for the existence of small presentations apply under the assumption that $(\mathcal{C} o f, \mathcal{W} \cap \mathcal{F} i b)$ is strictly cofibrantly generated. We do not know an example of a cofibrantly generated model category for which $(\mathcal{C} o f, \mathcal{W} \cap \mathcal{F} i b)$ fails to be strictly cofibrantly generated - such an example cannot be finitely generated.

More specifically, in Section 4, we obtain the following results (see Theorem 4.5). Let $(\mathcal{M}, \mathcal{C} o f, \mathcal{W}, \mathcal{F} i b)$ be a model category.

(A) Suppose that $(\mathcal{C} \circ f, \mathcal{W} \cap \mathcal{F} i b)$ is strictly cofibrantly generated. Then, assuming Vopěnka's principle, $\mathcal{M}$ admits a small presentation. Therefore $\mathcal{M}$ is Quillen equivalent to a combinatorial model category.

(B) Suppose that both weak factorization systems of $\mathcal{M}$ are perfectly cofibrantly generated. Then $\mathcal{M}$ admits a small presentation. Therefore $\mathcal{M}$ is Quillen equivalent to a combinatorial model category.

On the other hand:

(C) There are model categories whose weak factorization systems are weakly cofibrantly generated but they are not Quillen equivalent to a combinatorial model category. Moreover, even the homotopy category of such a model category is not equivalent to the homotopy category of a combinatorial model category in general.

The method we use to prove (A) and (B) follows Dugger's method [5] for proving that a combinatorial model category has a small presentation. Based on the preliminary results about cofibrant objects and weak equivalences in Section 3, we show that this method can be applied to more general model categories - assuming Vopěnka's principle or not. Concerning the role of the large cardinal axiom in Vopernka's principle, it is worth mentioning that this is used through several of its non-trivially equivalent forms or non-trivial implications and it is required at many steps in the proof of (A).

The results in this paper help to clarify the situation regarding the comparison between cofibrantly generated and combinatorial model categories, but aspects of this comparison and its precise set-theoretical status remain open. For example, we do not know if there is an example of a cofibrantly generated model category $(\mathcal{M}, \mathcal{C} o f, \mathcal{W}, \mathcal{F} i b)$ which does not have a small presentation. Moreover, we do not know if assuming the negation of Vopěnka's principle, there is such an example for which $(\mathcal{C} \circ f, \mathcal{W} \cap \mathcal{F} i b)$ is strictly cofibrantly generated.

The paper is organized as follows. Section 2 is concerned with the general properties of weak factorization systems: there we introduce and compare different notions of cofibrant generation. Section 3 collects some useful properties of strictly cofibrantly generated weak factorization systems. In particular, we prove that the category of cofibrant objects has a small (colimit-)dense subcategory (see Theorem 3.1) and explore the consequences of this property. Moreover, we give general conditions on a model 
category for the class of weak equivalences (between cofibrant objects) to be closed under large enough filtered colimits (Proposition 3.12). In Section 4, we first present a short review of Dugger's method [5] for the construction of small presentations as it applies to more general model categories. Then we deduce our main results about the existence of small presentations (Theorem 4.5 and Corollary 4.6). Lastly, in Subsection 4.3, we discuss an example of a weakly cofibrantly generated model category whose homotopy category is not equivalent to the homotopy category of a combinatorial model category. Finally, in Section 5, we discuss the mistake in the argument of $[\mathbf{1 6}]$ and correct it under additional assumptions.

\section{Acknowledgments}

The first author was partially supported by SFB 1085 - Higher Invariants, University of Regensburg. The second author was supported by the Grant Agency of the Czech Republic under the grant P201/12/G028.

\section{Generating classes of cofibrations}

In this section we review the standard definition of a cofibrantly generated weak factorization system and introduce some variations of this notion. We are mainly interested in a slightly stronger notion of a weak factorization system where the domains of the generating cofibrations satisfy a stronger smallness condition. This condition will be required in the proofs of our main results but it may also be of independent interest. It is suggested by the fat small object argument $[\mathbf{1 4}]$ in the same way that the standard smallness condition arises naturally from Quillen's small object argument.

\subsection{Small objects}

Let $\mathcal{M}$ be a cocomplete category and $\mathcal{X}$ a class of morphisms. The class $\operatorname{cell}(\mathcal{X})$ of $\mathcal{X}$-cellular morphisms is the smallest class of morphisms which contains $\mathcal{X}$ and is closed under transfinite compositions and pushouts along morphisms in $\mathcal{X}$. The class $\operatorname{cof}(\mathcal{X})$ of $\mathcal{X}$-cofibrations is the smallest class of morphisms which is in addition closed under retracts. Let $\mathcal{X} \square$ (resp. $\square \mathcal{X}$ ) denote the class of morphisms which have the right lifting property (resp. left lifting property) with respect to each morphism in $\mathcal{X}$. It is easy to see that every $\mathcal{X}$-cofibration has the left lifting property with respect to $\mathcal{X}^{\square}$, that is, $\operatorname{cof}(\mathcal{X}) \subseteq \square\left(\mathcal{X}^{\square}\right)$.

Given a poset $P$ and $x \in P, \downarrow x=\{y \in P \mid y \leqslant x\}$ denotes the initial segment associated with $x$. An element $x$ of a poset $P$ is isolated if the subposet

$$
\downarrow x=\{y \in P \mid y<x\}
$$

has a greatest element $x^{-}$which is called the predecessor of $x$. A non-isolated element, which is not a least element, is called a limit element. For a poset $P$, a diagram $D: P \rightarrow \mathcal{M}$ is called smooth if for every limit element $x \in P$, the diagram

$$
(D(y \rightarrow x): D y \rightarrow D x)_{y<x}
$$

defines a colimit cocone of the restriction of $D$ to the subposet $\downarrow \downarrow x$. 
A poset $P$ is well-founded if each non-empty subset of $P$ contains a minimal element. A poset $P$ is called good if it is well-founded and has a least element $\perp$. Note that every non-empty well-ordered set is good. For a regular cardinal $\lambda$, we say that a good poset $P$ is $\lambda$-good if the initial segment $\downarrow x$ has cardinality $<\lambda$ for each $x \in P$. A good diagram $D: P \rightarrow \mathcal{M}$ is a smooth diagram where $P$ is a good poset. Such a diagram is essentially determined by $D(\perp)$ and the morphisms $D\left(x^{-} \rightarrow x\right)$ for all isolated elements $x \in P$. These morphisms $D\left(x^{-} \rightarrow x\right): D\left(x^{-}\right) \rightarrow D(x)$ are the links of the diagram $D$. The composite of a good diagram $D: P \rightarrow \mathcal{M}$ is the canonical morphism $D(\perp) \rightarrow \operatorname{colim}_{P} D$.

An object $X \in \mathcal{M}$ is $\lambda$-small relative to $\mathcal{X}$ if $\mathcal{M}(X,-)$ preserves colimits of smooth $\lambda$-directed chains of $\mathcal{X}$-cellular morphisms. We say that $X$ is small relative to $\mathcal{X}$ if it is $\lambda$-small relative to $\mathcal{X}$ for some regular cardinal $\lambda$. This is the standard notion of smallness that appears in the definition of cofibrantly generated model categories and it is suggested by the small object argument. This argument was originally used by Quillen in [15] and it was later formalized in the following way.

Theorem 2.1 (Small object argument). Let $\mathcal{M}$ be a cocomplete category and $\mathcal{X}$ a set of morphisms. Suppose that the domains of the morphisms in $\mathcal{X}$ are small relative to $\mathcal{X}$. Then every morphism $f$ in $\mathcal{M}$ admits a functorial factorization $f=p i$ into an $\mathcal{X}$-cellular morphism $i \in \operatorname{cell}(\mathcal{X})$ followed by a morphism $p \in \mathcal{X}^{\square}$.

Proof. See [10, Theorem 2.1.14].

Remark 2.2. If the domains of the morphisms in (the set) $\mathcal{X}$ are small relative to $\mathcal{X}$, then the small object argument together with the retract argument imply that $\operatorname{cof}(\mathcal{X})={ }^{\square}\left(\mathcal{X}^{\square}\right)($ see $[\mathbf{1 0}, 2.1 .15])$.

We will consider the following variation of the notion of smallness.

Definition 2.3. Let $\mathcal{M}$ be a category, $\mathcal{X}$ a set of morphisms, and $\lambda$ a regular cardinal. An object $X$ in $\mathcal{M}$ is called strictly $\lambda$-small relative to $\mathcal{X}$ if $\mathcal{M}(X,-)$ preserves $\lambda$ directed good colimits whose links are $\mathcal{X}$-cellular morphisms. We say that $X$ is strictly small relative to $\mathcal{X}$ if it is strictly $\lambda$-small for some regular cardinal $\lambda$.

Remark 2.4. In Definition 2.3, it is possible to restrict to $\lambda$-directed good colimits whose links are obtained as pushouts of morphisms in $\mathcal{X}$ as this leads to an equivalent notion. The equivalence can be demonstrated by replacing the $\mathcal{X}$-cellular links of a diagram by directed chains whose links are pushouts along morphisms in $\mathcal{X}$. An analogous statement is also true and well known for the definition of small objects.

We note that the composite of a good diagram whose links are $\mathcal{X}$-cellular morphisms has the left lifting property with respect to $\mathcal{X}^{\square}$ (see the proof of $[\mathbf{1 4}$, Proposition 4.4] or [11, Lemma A.1.5.6]). A strictly $\lambda$-small object is clearly $\lambda$-small. Therefore, a set of morphisms $\mathcal{X}$ whose domains are strictly small with respect to $\mathcal{X}$ permits the small object argument. Moreover, it also permits the fat small object argument $[\mathbf{1 4}]$ as stated below (see also [11, A.1.5]).

We first recall some notation from $[\mathbf{1 4}]$. Let $\operatorname{Po}(\mathcal{X})$ denote the class of morphisms which are obtained as pushouts along a morphism in $\mathcal{X}$. Let $\operatorname{GdDirPo}_{\lambda}(\mathcal{X})$ denote the class of morphisms which are obtained as composites of $\lambda$-good $\lambda$-directed diagrams whose links are in $\operatorname{Po}(\mathcal{X})$. 
Theorem 2.5 (Fat small object argument). Let $\mathcal{M}$ be a cocomplete category, $\mathcal{X}$ a set of morphisms, and $\lambda$ a regular cardinal. Suppose that the domains of the morphisms in $\mathcal{X}$ are strictly $\lambda$-small relative to $\mathcal{X}$. Then every morphism $f$ in $\mathcal{M}$ admits a factorization $f=p i$ into a morphism $i \in \operatorname{GdDirPo}_{\lambda}(\mathcal{X})$ followed by a morphism $p \in \mathcal{X}^{\square}$.

Proof. This factorization can be obtained using a variation of the small object argument as in [14, Theorem A.1]. Alternatively, the small object argument yields a functorial factorization $f=p i$ where $i$ is an $\mathcal{X}$-cellular morphism and $p \in \mathcal{X}^{\square}$. Then the proof of [14, Theorem 4.11] applies to show that $i \in \operatorname{GdDirPo}_{\lambda}(\mathcal{X})$ as required (see also [14, Remark A.2]).

Remark 2.6. Any directed poset $P$ contains a cofinal good subposet $Q$ (see, e.g., [8, 2.7.2]). If $P$ is $\lambda$-directed, then $Q$ is $\lambda$-directed too. But, given a $\lambda$-directed diagram $P \rightarrow \mathcal{M}$, its restriction to $Q$ does not need to be smooth. So we do not know whether, for a strictly $\lambda$-small object $X$, the functor $\mathcal{M}(X,-)$ preserves $\lambda$-directed colimits of $\mathcal{X}$-cellular morphisms in general.

\subsection{Weak factorization systems}

We recall that a weak factorization system $(\mathcal{L}, \mathcal{R})$ in a category $\mathcal{M}$ consists of two classes of morphisms $\mathcal{L}$ and $\mathcal{R}$ in $\mathcal{M}$ such that

(1) $\mathcal{R}=\mathcal{L}^{\square}, \mathcal{L}={ }^{\square} \mathcal{R}$,

(2) every morphism $h$ of $\mathcal{M}$ has a factorization $h=g f$ with $f \in \mathcal{L}$ and $g \in \mathcal{R}$.

The small object argument provides examples of weak factorization systems in general cocomplete categories. One feature of these weak factorization systems is that they are determined by a set of morphisms.

Definition 2.7. Let $(\mathcal{L}, \mathcal{R})$ be a weak factorization system in $\mathcal{M}$. A set of morphisms $\mathcal{X} \subseteq \mathcal{L}$ is called a generator for $\mathcal{R}$ if $\mathcal{X}^{\square}=\mathcal{R}$.

This property isolates one of the requirements for the weak factorization systems in the definition of a cofibrantly generated model category. A generator $\mathcal{X}$ for $\mathcal{R}$ obviously determines $\mathcal{R}$, and therefore $\mathcal{L}$, in terms of lifting properties. As the following remark shows, this notion generalizes the standard notion of a strong generator in a category.

Remark 2.8. Let $\mathcal{M}$ be a category with finite coproducts and equalizers and consider the trivial (weak) factorization system $(\mathcal{M} \rightarrow$, Iso $\mathcal{M})$ which is defined by the classes of all morphisms and isomorphisms in $\mathcal{M}$, respectively. We claim that this weak factorization system has a generator if and only if $\mathcal{M}$ has a strong generator.

Suppose that $\mathcal{A}$ is a strong generator of $\mathcal{M}$. Under the assumption on equalizers, this means that a morphism $f: X \rightarrow Y$ is an isomorphism if $\mathcal{M}(A, f): \mathcal{M}(A, X) \rightarrow$ $\mathcal{M}(A, Y)$ is a bijection for all $A \in \mathcal{A}$. Consider the set $\mathcal{X}$ which consists of the morphisms

$$
0_{A}: 0 \rightarrow A \text { and } \nabla_{A}: A \sqcup A \rightarrow A,
$$

for all $A \in \mathcal{A}$ where 0 denotes an initial object and $\nabla_{A}$ is the codiagonal. A morphism $f: X \rightarrow Y$ has the right lifting property with respect to $0_{A}\left(\right.$ resp. $\left.\nabla_{A}\right)$ if and only if 
the map

$$
\mathcal{M}(A, f): \mathcal{M}(A, X) \rightarrow \mathcal{M}(A, Y)
$$

is surjective (resp. injective). Hence $f \in \mathcal{X}^{\square}$ if and only if $\mathcal{M}(A, f)$ is bijective for all $A \in \mathcal{A}$.

For the converse, suppose that $\mathcal{X} \subseteq \mathcal{M}^{\rightarrow}$ is a generator for Iso $_{\mathcal{M}}$. Consider the set $\mathcal{A}$ of domains and codomains of the morphisms in $\mathcal{X}$. It is easy to see that a morphism $f: X \rightarrow Y$ such that $\mathcal{M}(A, f)$ is bijective for all $A \in \mathcal{A}$ also has the right lifting property with respect to $\mathcal{X}$, and therefore it is an isomorphism.

Combined with the notions of smallness above, we have the following hierarchy of notions of cofibrant generation for weak factorization systems.

Definition 2.9. Let $(\mathcal{L}, \mathcal{R})$ be a weak factorization system in a cocomplete category $\mathcal{M}$ and $\lambda$ a regular cardinal.

(a) $(\mathcal{L}, \mathcal{R})$ is called setwise cofibrantly generated if there is a generator $\mathcal{X} \subseteq \mathcal{L}$ for $\mathcal{R}$.

(b) $(\mathcal{L}, \mathcal{R})$ is called weakly cofibrantly generated if there is a generator $\mathcal{X} \subseteq \mathcal{L}$ for $\mathcal{R}$ such that $\mathcal{L}=\operatorname{cof}(\mathcal{X})$. In this case, $\mathcal{X}$ is called a generating set for $(\mathcal{L}, \mathcal{R})$.

(c) $(\mathcal{L}, \mathcal{R})$ is called $(\lambda-)$ cofibrantly generated if there is a generator $\mathcal{X} \subseteq \mathcal{L}$ for $\mathcal{R}$ such that the domains of the morphisms in $\mathcal{X}$ are $(\lambda$-)small relative to $\mathcal{X}$.

(d) $(\mathcal{L}, \mathcal{R})$ is called strictly $(\lambda$-) cofibrantly generated if there is a generator $\mathcal{X} \subseteq \mathcal{L}$ for $\mathcal{R}$ such that the domains of the morphisms in $\mathcal{X}$ are strictly $(\lambda$-)small relative to $\mathcal{X}$.

(e) $(\mathcal{L}, \mathcal{R})$ is called semi-perfectly $(\lambda$-) cofibrantly generated if there is a generator $\mathcal{X} \subseteq \mathcal{L}$ for $\mathcal{R}$ such that the domains of the morphisms in $\mathcal{X}$ are $(\lambda$-)presentable objects in $\mathcal{M}$.

(f) $(\mathcal{L}, \mathcal{R})$ is called perfectly $(\lambda$-) cofibrantly generated if there is a generator $\mathcal{X} \subseteq \mathcal{L}$ for $\mathcal{R}$ such that the domains and the codomains of the morphisms in $\mathcal{X}$ are $(\lambda-)$ presentable objects in $\mathcal{M}$.

The definition in (c) corresponds to the standard notion that appears in the definition of cofibrantly generated model categories. The weaker notion in (b) was considered in $[\mathbf{1 9}]$ together with the corresponding weaker notion of a cofibrantly generated model category. Concerning the definition in (a), note that a generator $\mathcal{X}$ does not permit the small object argument in general, and therefore we cannot identify $\mathcal{L}$ with $\operatorname{cof}(\mathcal{X})$. We are mainly interested in $(\mathrm{d})$ which is motivated by the fat small object argument as previously explained. We have the following obvious implications:

perfectly cof. generated $\Longrightarrow$ strictly cof. generated $\Longrightarrow$ cofibrantly generated<smiles>C1=CC=C1</smiles>

weakly cof. generated<smiles>C1=CC=C1</smiles>

setwise cof. generated 
Example 2.10. Let $\mathcal{K}$ be the category of compact Hausdorff spaces with the trivial weak factorization system $\left(\mathcal{K} \rightarrow\right.$, Iso $\left._{\mathcal{K}}\right)$. Since the one-point space $*$ is a strong generator in $\mathcal{K}$, this weak factorization system has a generator $\mathcal{X}$ which consists of the maps (see Remark 2.8)

$$
0: \varnothing \rightarrow\{*\} \text { and } \nabla:\{*\} \sqcup\{*\} \rightarrow\{*\} .
$$

Moreover, it is weakly cofibrantly generated because every morphism in $\mathcal{K}$ is in $\operatorname{cof}(\mathcal{X})$. To see this, note first that every surjective map can be obtained as a pushout along a coproduct of copies of the map $\nabla$. For an injective map $f: X \rightarrow Y$, consider the factorization

$$
X \stackrel{f}{\cong} f(X) \stackrel{j}{\longrightarrow} f(X) \sqcup \mathbb{F}\left(Y-f(X)^{\delta}\right) \stackrel{p}{\longrightarrow} Y,
$$

where $(Y-f(X))^{\delta}$ denotes the discrete topological space with underlying set $Y-$ $f(X)$ and $\mathbb{F}$ is the associated free compact Hausdorff space. Recall that for a set $Z$, $\mathbb{F}(Z)$ is the compact Hausdorff space of all ultrafilters on $Z$. The map $j$ is clearly in $\operatorname{cell}(\mathcal{X})$, by definition. The map $p$ is surjective, so it is in $\operatorname{cof}(\mathcal{X})$, too. Note that in this case we have $\operatorname{cell}(\mathcal{X})=\operatorname{cof}(\mathcal{X})$.

However, this weak factorization system is not cofibrantly generated because $\varnothing$ is the only small object in $\mathcal{K}$. To see this, it suffices to show that $*$ is not small in $\mathcal{K}$. A regular cardinal $\lambda$ can be written as the colimit of the $\lambda$-directed chain of all ordinals with cardinality less than $\lambda$. Then an ultrafilter on $\lambda$ which contains the filter of all subsets whose complement has cardinality less than $\lambda$ defines a point in $\mathbb{F}(\lambda)$ which does not factor through any stage of the chain. Hence, $*$ is not $\lambda$-small in $\mathcal{K}$.

We do not know an example of a cofibrantly generated weak factorization system which is not strictly cofibrantly generated. Such an example does not exist in the case of $\aleph_{0}$ by the following result.

Proposition 2.11. Let $(\mathcal{L}, \mathcal{R})$ be a cofibrantly generated weak factorization system in $\mathcal{M}$ generated by a set of morphisms $\mathcal{X}$ whose domains are $\aleph_{0}$-small relative to $\mathcal{X}$. Then $(\mathcal{L}, \mathcal{R})$ is strictly $\aleph_{0}$-cofibrantly generated.

Proof. It suffices to show that any domain $X$ of a morphism from $\mathcal{X}$ is strictly $\aleph_{0^{-}}$ small. Let $P$ be a directed good poset and $D: P \rightarrow \mathcal{M}$ a good diagram whose links are $\mathcal{X}$-cellular morphisms. Following $[\mathbf{1}, 1.6]$, we can express $P$ as a union of a smooth chain of directed subposets $P_{k}$ each of which has cardinality less than that of $P$. Let $D_{k}$ denote the restriction of $D$ to $\downarrow P_{k}$. Here $\downarrow P_{k}$ denotes the initial segment generated by $P_{k}$. Note that $P_{k}$ is cofinal in $\downarrow P_{k}$. Moreover, $D_{k}$ is again a good diagram whose links are in $\operatorname{cell}(\mathcal{X})$. Following [14, Remark 4.8], the induced morphisms for $k<k^{\prime}$,

$$
\operatorname{colim} D_{k} \rightarrow \operatorname{colim} D_{k^{\prime}}
$$

are $\mathcal{X}$-cellular. Clearly, we have $\operatorname{colim}_{k} \operatorname{colim}_{\downarrow P_{k}} D_{k} \cong \operatorname{colim}_{P} D$. Then we can proceed by transfinite induction on the cofinality of $P$, that is, the smallest cardinality for which there is a cofinal subposet (cf. [1, Corollary 1.7]). 


\section{Cofibrant objects and weak equivalences}

Let $\mathcal{M}$ be a cocomplete category and $(\mathcal{L}, \mathcal{R})$ a weak factorization system in $\mathcal{M}$. An object $X$ in $\mathcal{M}$ is called $\mathcal{L}$-cofibrant, or simply cofibrant, if the morphism $0 \rightarrow X$ is in $\mathcal{L}$, where 0 denotes an initial object. Let $\mathcal{M}_{c} \subseteq \mathcal{M}$ denote the full subcategory which is spanned by the cofibrant objects.

In this section, we discuss some useful properties of the full subcategory $\mathcal{M}_{c} \subseteq \mathcal{M}$ in the case where $(\mathcal{L}, \mathcal{R})$ is strictly cofibrantly generated. We will also use these properties to identify general conditions on a model category so that the class of weak equivalences (between cofibrant objects) is closed under large enough filtered colimits. The results in this section form the technical backbone of the proof of our main theorem in the next section (Theorem 4.5).

\subsection{Small dense subcategories of cofibrant objects}

Let $\mathcal{M}$ be a cocomplete category and $(\mathcal{L}, \mathcal{R})$ a strictly cofibrantly generated weak factorization system in $\mathcal{M}$ with a generating set $\mathcal{X}$. For any cardinal $\lambda$, let $\widetilde{\mathcal{A}}(\mathcal{X}, \lambda)$ be the smallest full subcategory of $\mathcal{M}$ which contains the domains and codomains of the morphisms in $\mathcal{X}$ and is closed under $\lambda$-small colimits in $\mathcal{M}$. Here $\lambda$-small refers to colimits of diagrams indexed by categories whose sets of morphisms have cardinality smaller than $\lambda$. We consider the small full subcategory $\mathcal{A}(\mathcal{X}, \lambda)$ of $\mathcal{M}_{c}$ given by

$$
\mathcal{A}(\mathcal{X}, \lambda)=\widetilde{\mathcal{A}}(\mathcal{X}, \lambda) \cap \mathcal{M}_{c}
$$

Note that the construction of $\mathcal{A}(\mathcal{X}, \lambda)$ has the following properties:

(a) If $\lambda \leqslant \mu$, then $\mathcal{A}(\mathcal{X}, \lambda) \subseteq \mathcal{A}(\mathcal{X}, \mu)$.

(b) If $\lambda=\bigvee_{i<\alpha} \lambda_{i}$, then $\mathcal{A}(\mathcal{X}, \lambda)=\bigcup_{i<\alpha} \mathcal{A}\left(\mathcal{X}, \lambda_{i}\right)$.

In the following statements, we will make use of the definition and the properties of preaccessible categories (see $[\mathbf{2}]$ ). For a regular cardinal $\lambda$, a category $\mathcal{C}$ is called $\lambda$-preaccessible if it has a set of $\lambda$-presentable objects $\mathcal{A}$ such that each object in $\mathcal{C}$ is a $\lambda$-filtered colimit of objects in $\mathcal{A}$. Note that we do not assume that $\mathcal{C}$ has all $\lambda$ filtered colimits. We say that $\mathcal{C}$ is preaccessible if it is $\lambda$-preaccessible for some regular cardinal $\lambda$. A preaccessible category has a small dense subcategory [2]. Conversely, assuming Vopěnka's principle, a category which has a small dense subcategory is preaccessible [2, Theorem 1].

Recall also that a full subcategory $\mathcal{A}$ of a category $\mathcal{C}$ is colimit-dense provided that every object of $\mathcal{C}$ is a colimit of a diagram in $\mathcal{A}$. Assuming Vopěnka's principle, a category which has a small colimit-dense subcategory has also a small dense subcategory [1, Theorem 6.35].

Theorem 3.1. Let $\mathcal{M}$ be a cocomplete category and $(\mathcal{L}, \mathcal{R})$ a strictly $\kappa$-cofibrantly generated weak factorization system in $\mathcal{M}$ with generating set $\mathcal{X}$.

(1) For every regular cardinal $\lambda \geqslant \kappa$, every object of $\mathcal{M}_{c}$ is a $\lambda$-filtered colimit in $\mathcal{M}$ of objects in $\mathcal{A}(\mathcal{X}, \lambda)$.

(2) Suppose that $(\mathcal{L}, \mathcal{R})$ is perfectly $\kappa$-cofibrantly generated with generating set $\mathcal{X}$ and let $\lambda \geqslant \kappa$ be a regular cardinal. Then the small colimit-dense subcategory $\mathcal{A}(\mathcal{X}, \lambda) \subseteq \mathcal{M}_{c}$ consists of $\lambda$-presentable objects in $\mathcal{M}$. In particular, every cofibrant object is presentable in $\mathcal{M}$. 
(3) Assuming Vopěnka's principle, $\mathcal{M}_{c}$ is preaccessible and therefore every cofibrant object is presentable in $\mathcal{M}_{c}$. Moreover, there are arbitrarily large regular cardinals $\lambda$ such that every object of $\mathcal{A}(\mathcal{X}, \lambda)$ is $\lambda$-presentable in $\mathcal{M}_{c}$.

Proof. First note that the domains of the morphisms in $\mathcal{X}$ are strictly $\lambda$-small for each $\lambda \geqslant \kappa$. Following the proof of [14, Theorem 4.11] (cf. Theorem 2.5), every $\mathcal{X}$ cellular object is a $\lambda$-good $\lambda$-directed colimit of a diagram in $\mathcal{M}$ consisting of objects in $\mathcal{A}(\mathcal{X}, \lambda)$ and $\mathcal{X}$-cellular morphisms. Then every cofibrant object is a $\lambda$-directed colimit in $\mathcal{M}$ of objects from $\mathcal{A}(\mathcal{X}, \lambda)$ (see the proof of $[\mathbf{1 4}$, Corollary 5.1] and [13, Proposition 2.3.11]). This completes the proof of (1). (2) follows from the construction of the small colimit-dense subcategory $\mathcal{A}(\mathcal{X}, \lambda)$. The presentability of the cofibrant objects can also be seen directly from the small object argument.

The first part of (3) follows from (1) and [1, Theorem 6.35] combined with [2, Theorem 1] or [1, Corollary 6.11]. For the second part, we may assume that $\mathcal{M}_{c}$ is a full subcategory of the category of graphs Gra because $\mathcal{M}_{c}$ has a small dense subcategory (see [1, Proposition 1.26, Theorem 2.65]). Assuming Vopěnka's principle, the inclusion $\mathcal{M}_{c} \rightarrow$ Gra preserves $\lambda_{0}$-directed colimits for some $\lambda_{0}$ by $[\mathbf{1}$, Theorem 6.9]. For $X \in \mathcal{M}_{c},|X|$ denotes the cardinality of the underlying set of the graph $X$. Then, given a regular cardinal $\alpha \geqslant \lambda_{0},|X|<\alpha$ implies that $X$ is $\alpha$-presentable in $\mathcal{M}_{c}$ because it is so in Gra. For any cardinal $\alpha$, let $\alpha^{*} \geqslant \alpha$ be the smallest cardinal such that for each $X \in \mathcal{A}(\mathcal{X}, \alpha)$, we have that $|X|<\alpha^{*}$. Let $\mathbf{C}$ be the class of all cardinals $\alpha \geqslant \lambda_{0}$ such that $\alpha=\alpha^{*}$. As in the proof of [2, Theorem 1], we easily check that the class $\mathbf{C}$ is closed and unbounded. Since the class of all regular cardinals is stationary under Vopěnka's principle, it follows that $\mathbf{C}$ contains a regular cardinal $\lambda$ (see $[\mathbf{1 2}$, Theorem 3]). This regular cardinal $\lambda$ satisfies (3). Since $\lambda_{0}$ can be chosen to be arbitrarily large, the same holds for $\lambda$, too.

Remark 3.2. In Theorem 3.1(2), the objects of $\mathcal{A}(\mathcal{X}, \lambda)$ are $\lambda$-presentable in $\mathcal{M}$ by construction. We cannot claim that they are $\lambda$-presentable in $\mathcal{M}_{c}$ because the inclusion $\mathcal{M}_{c} \rightarrow \mathcal{M}$ need not preserve $\lambda$-directed colimits.

The following statement is well known in the context of combinatorial model categories (cf. [5, Proposition 7.2]) and will be useful in the proofs of our main results. We state it here more generally for weak factorization systems.

Corollary 3.3. Let $\mathcal{M}$ be a cocomplete category and $(\mathcal{L}, \mathcal{R})$ a strictly cofibrantly generated weak factorization system.

(1) Assuming Vopěnka's principle, for arbitrarily large regular cardinals $\lambda$, the following holds: every morphism $f: X \rightarrow Y$ between $\lambda$-presentable objects in $\mathcal{M}_{c}$ admits a functorial factorization $X \stackrel{i}{\rightarrow} Z \stackrel{p}{\rightarrow} Y$ with $i \in \mathcal{L}, p \in \mathcal{R}$ and $Z$ is again $\lambda$-presentable in $\mathcal{M}_{c}$.

(2) Suppose that $(\mathcal{L}, \mathcal{R})$ is perfectly cofibrantly generated. Then for arbitrarily large regular cardinals $\lambda$, the following holds: every morphism $f: X \rightarrow Y$ between cofibrant objects which are $\lambda$-presentable in $\mathcal{M}$ admits a functorial factorization $X \stackrel{i}{\rightarrow} Z \stackrel{p}{\rightarrow} Y$ with $i \in \mathcal{L}, p \in \mathcal{R}$ and $Z \in \mathcal{M}_{c}$ is again $\lambda$-presentable in $\mathcal{M}$.

Proof. We first prove (2) whose proof is based on the small object argument and is similar to the combinatorial case. Let $\mathcal{X} \subseteq \mathcal{L}$ be a generator for $\mathcal{R}$ such that the 
domains and codomains of $\mathcal{X}$ are $\mu$-presentable. For every morphism $f: X \rightarrow Y$ in $\mathcal{M}$ form the colimit $F_{0} f$ of the diagram

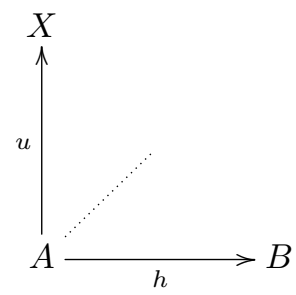

consisting of all spans $(u, h)$ with $h: A \rightarrow B$ in $\mathcal{X}$, one for each commutative square

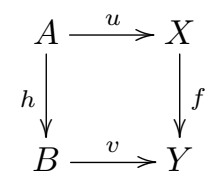

Let $\alpha_{0 f}: X \rightarrow F_{0} f$ denote the component of the colimit cocone (the other components are morphisms $B \rightarrow F_{0} f$ and the squares

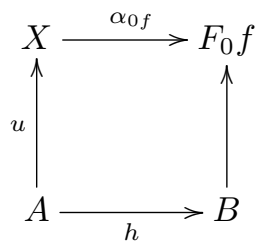

are commutative). Let $\beta_{0 f}: F_{0} f \rightarrow Y$ be the morphism induced by the morphisms $f$ and $v$ 's. This way we get a functor $F_{0}: \mathcal{M}^{\rightarrow} \rightarrow \mathcal{M}$ which preserves $\mu$-filtered colimits. Let $F_{i} f, \alpha_{i f}$ and $\beta_{i f}, i \leqslant \mu$, be given by the following transfinite induction:

$$
F_{i+1} f=F_{0} \beta_{i f}, \quad \alpha_{i+1, f}=\alpha_{0, \beta_{i f}} \alpha_{i f}, \quad \beta_{i+1, f}=\beta_{0, \beta_{i f}}
$$

and the limit step is given by taking colimits. Then the functor $F_{\mu}: \mathcal{M} \rightarrow \rightarrow \mathcal{M}$ preserves $\mu$-filtered colimits. For each $f: X \rightarrow Y$, the resulting functorial factorization

$$
X \stackrel{i}{\rightarrow} F_{\mu}(f) \stackrel{p}{\rightarrow} Y
$$

has $i \in \mathcal{L}$ and $p \in \mathcal{R}$ by the small object argument. The existence of $\lambda$ can be argued similarly to $[\mathbf{1}$, Theorem 2.19] whose proof is also valid in this context (see Proposition 3.6 below for a suitable formulation). Indeed, it suffices to check that $[\mathbf{1}$, Remark 2.15] can be used. In other words, it suffices to show that every morphism between cofibrant objects is a $\mu$-directed colimit of morphisms between cofibrant objects which are $\mu$-presentable in $\mathcal{M}$. This is guaranteed by Theorem 3.1(2) applied to the weak factorization system on $\mathcal{M}^{\rightarrow}$ where the cofibrations are defined pointwise - this is again perfectly $\mu$-cofibrantly generated.

The proof of (1) starts similarly. Let $\mathcal{X} \subseteq \mathcal{L}$ be a generator for $\mathcal{R}$ such that the domains of $\mathcal{X}$ are strictly $\mu$-small relative to $\mathcal{X}$. For every morphism $f: X \rightarrow Y$ in $\mathcal{M}_{c}$, we can proceed as before and define a functor $F_{\mu}: \mathcal{M}_{c} \rightarrow \mathcal{M}_{c}$. But we will need to use a different approach in order to obtain $\lambda$. 
By Theorem 3.1(3), the category $\mathcal{M}_{c}$ is preaccessible. As in the proof of Theorem 3.1(3), we may assume that $\mathcal{M}_{c}$ is a full subcategory of the category of graphs Gra such that the inclusion $\mathcal{M}_{c} \rightarrow$ Gra preserves $\lambda_{0}$-directed colimits for some $\lambda_{0}$. For $X \in \mathcal{M}_{c},|X|$ denotes the cardinality of the underlying set of the graph $X$. Given a regular cardinal $\alpha \geqslant \lambda_{0},|X|<\alpha$ implies that $X$ is $\alpha$-presentable in $\mathcal{M}_{c}$. The proof of $\left[\mathbf{2}\right.$, Theorem 1] considered a closed and unbounded class of cardinals $\mathbf{C}_{1}$ with the property that for each $\alpha \in \mathbf{C}_{1}$, every subobject $Y \subseteq X$ in Gra with $X \in \mathcal{M}_{c}$ and $|Y|<\alpha$ can be embedded into a strong subobject $Y^{*} \subseteq X$ in $\mathcal{M}_{c}$ with $\left|Y^{*}\right|<\alpha$. Assuming Vopěnka's principle, it follows that there is a regular cardinal $\lambda$ in $\mathbf{C}_{1}$ (see the proof of [2, Theorem 1]). Given such a regular cardinal $\lambda$, each object $X \in \mathcal{M}_{c}$ is a $\lambda$-directed colimit of objects in $\mathcal{M}_{c}$ with cardinality (as graphs) $<\lambda$. Thus, if $X$ is $\lambda$-presentable object in $\mathcal{M}_{c}$, then it is a split subobject of an object with cardinality $<\lambda$, and therefore also $|X|<\lambda$.

For any cardinal $\alpha$, let $\alpha^{*} \geqslant \alpha$ be the smallest cardinal such that $|X|,|Y|<\alpha$ implies that $\left|F_{\mu}(f)\right|<\alpha^{*}$ for every $f: X \rightarrow Y$, where $X \rightarrow F_{\mu}(f) \rightarrow Y$ is the factorization constructed above. Let $\mathbf{C}_{2}$ be the class of all cardinals $\alpha \geqslant \lambda_{0}$ such that $\alpha=\alpha^{*}$. As in the proof of [2, Theorem 1], we easily check that the class $\mathbf{C}_{2}$ is closed and unbounded. The intersection $\mathbf{C}_{3}=\mathbf{C}_{1} \cap \mathbf{C}_{2}$ is again closed and unbounded. Since the class of all regular cardinals is stationary under Vopěnka's principle (see [12, Theorem 3]), it follows that $\mathbf{C}_{3}$ contains a regular cardinal $\lambda$, and this has the required property. Since $\lambda_{0}$ can be chosen to be arbitrarily large, the same applies to $\lambda$, too.

Corollary 3.4. Let $(\mathcal{M}, \mathcal{C} o f, \mathcal{W}, \mathcal{F} i b)$ be a model category. Suppose that the weak factorization system $(\mathcal{C} o f, \mathcal{W} \cap \mathcal{F} i b)$ is strictly $\kappa$-cofibrantly generated with generating set $\mathcal{X}$. Then:

(1) For every regular cardinal $\lambda \geqslant \kappa$, every object of $\mathcal{M}_{c}$ is a $\lambda$-filtered colimit of objects in $\mathcal{A}(\mathcal{X}, \lambda)$.

(2) Suppose that $(\mathcal{C} o f, \mathcal{W} \cap \mathcal{F} i b)$ is perfectly $\kappa$-cofibrantly generated with generating set $\mathcal{X}$ and let $\lambda \geqslant \kappa$ be a regular cardinal. Then the small colimit-dense subcategory $\mathcal{A}(\mathcal{X}, \lambda) \subseteq \mathcal{M}_{c}$ consists of $\lambda$-presentable objects in $\mathcal{M}$. In particular, every cofibrant object is presentable in $\mathcal{M}$.

(3) Assuming Vopěnka's principle, $\mathcal{M}_{c}$ is preaccessible and therefore every cofibrant object is presentable in $\mathcal{M}_{c}$. Moreover, there are arbitrarily large regular cardinals $\lambda$ such that every object of $\mathcal{A}(\mathcal{X}, \lambda)$ is $\lambda$-presentable in $\mathcal{M}_{c}$.

(4) Suppose that every object is cofibrant and $(\mathcal{C}$ of $\cap \mathcal{W}, \mathcal{F}$ ib) is setwise cofibrantly generated. Then, assuming Vopěnka's principle, $\mathcal{M}$ is a combinatorial model category.

Proof. (1)-(3) are special cases of Theorem 3.1. For (4), Theorem 3.1 yields that $\mathcal{M}=\mathcal{M}_{c}$ is locally presentable and every object is presentable. In particular, the weak factorization system $(\mathcal{C} \circ f \cap \mathcal{W}, \mathcal{F} i b)$ is cofibrantly generated.

Remark 3.5. By combining the arguments in Corollaries 3.3(1) and 3.4(3) (or by applying Proposition 3.7 below), it follows similarly that there are arbitrarily large regular cardinals $\lambda$ for which both Corollary 3.3(1) for $(\mathcal{L}, \mathcal{R})=(\mathcal{C} \circ f, \mathcal{W} \cap \mathcal{F} i b)$ and Corollary 3.4(3) are satisfied. 


\subsection{Functors between preaccessible categories}

We digress slightly to add some comments on the arguments that were used in the proofs of the previous subsection. The comments concern certain variations of the Uniformization Theorem for accessible categories [1, Theorem 2.19] and may be of independent interest too.

First we introduce some terminology which is inspired from the context of Theorem 3.1. Given a category $\mathcal{M}$ and a full subcategory $\mathcal{B} \subseteq \mathcal{M}$, we denote by $\operatorname{Pres}_{\lambda}^{\mathcal{M}}(\mathcal{B})$ the full subcategory of $\mathcal{B}$ spanned by those objects which are $\lambda$-presentable in $\mathcal{M}$. If $\mathcal{M}$ admits $\kappa$-filtered colimits, we say that $\mathcal{B}$ is $\kappa$-preaccessible in $\mathcal{M}$ if there is a small full subcategory $\mathcal{A} \subseteq \mathcal{B}$ which consists of $\kappa$-presentable objects in $\mathcal{M}$ such that every object in $\mathcal{B}$ is a $\kappa$-filtered colimit in $\mathcal{M}$ of objects that lie in $\mathcal{A}$.

The following result can be regarded as a kind of "relative" Uniformization Theorem.

Proposition 3.6. Let $\mathcal{M}$ and $\mathcal{N}$ be categories which admit $\kappa$-filtered colimits for some regular cardinal $\kappa$, and

$$
F_{i}: \mathcal{M} \rightarrow \mathcal{N}, \quad i \in I,
$$

a small collection of functors which preserve $\kappa$-filtered colimits. Let $\mathcal{B} \subseteq \mathcal{M}$ be a full subcategory which is $\kappa$-preaccessible in $\mathcal{M}$ and suppose that for every $X \in \mathcal{B}, F_{i}(X) \in$ $\mathcal{N}$ is presentable in $\mathcal{N}$ for every $i \in I$. Then there are arbitrarily large regular cardinals $\lambda$ such that for every $X \in \operatorname{Pres}_{\lambda}^{\mathcal{M}}(\mathcal{B}), F_{i}(X)$ is $\lambda$-presentable in $\mathcal{N}$ for every $i \in I$.

Proof. The proof follows [1, Theorem 2.19, Remark 2.15]. Let $\mathcal{A} \subseteq \mathcal{B}$ be a small full subcategory of $\kappa$-presentable objects in $\mathcal{M}$ such that every object in $\mathcal{B}$ is a $\kappa$-directed colimit of objects from $\mathcal{A}$. Let $\kappa_{1} \triangleright \kappa$ be a regular cardinal such that for each $X \in \mathcal{A}$, $F_{i}(X) \in \mathcal{N}$ is $\kappa_{1}$-presentable in $\mathcal{N}$ for all $i \in I$. We claim that each $\lambda \triangleright \kappa_{1}$ has the required property. We recall that for each regular cardinal $\mu$, we have $\mu \triangleleft \mu^{+}$where $\mu^{+}$denotes the cardinal successor of $\mu$. We refer to $[\mathbf{1}, 2.11-2.13]$ for the definition and properties of the relation $\triangleright$.

Let $X \in \mathcal{B}$ be $\lambda$-presentable in $\mathcal{M}$. By assumption, $X$ is a $\kappa$-directed colimit of a diagram $D: P \rightarrow \mathcal{M}$ whose values are in $\mathcal{A}$. We consider the new poset $\hat{P}$ of all $\kappa$-directed $\lambda$-small subsets of $P$ ordered by inclusion. Then $\hat{P}$ is $\lambda$-directed. We define a new diagram $\hat{D}: \hat{P} \rightarrow \mathcal{M}$ which sends a subset $Q \subseteq P$ to the colimit in $\mathcal{M}$ of the restriction of $D$ to $Q$. This defines a $\lambda$-directed diagram $\hat{D}$ whose colimit is again $X$. As a consequence, the identity of $X$ factors through some stage of this diagram $\hat{D}$ and therefore $X$ is a split subobject of a $\lambda$-small $\kappa$-directed colimit of objects in $\mathcal{A}$. For each $i \in I, F_{i}(X)$ is then a split subobject of a $\lambda$-small $\kappa$-directed colimit of $\kappa_{1}$-presentable objects in $\mathcal{N}$. Therefore $F_{i}(X)$ is $\lambda$-presentable for every $i \in I$, as required.

Secondly, the argument that was used in the proofs of Theorem 3.1(3) and Corollary 3.3(1) generalizes to obtain the following more general result about functors between preaccessible categories under the assumption of Vopěnka's principle. It is an analogue in this context of the Uniformization Theorem for accessible functors (see [1, Theorem 2.19]).

Proposition 3.7. Let $F_{i}: \mathcal{M}_{i} \rightarrow \mathcal{N}, i \in I$, be a small collection of functors between preaccessible categories $\mathcal{M}_{i}, i \in I$, and $\mathcal{N}$. Assume that Vopěnka's principle holds. 
Then there are arbitrarily large regular cardinals $\lambda$ such that each functor $F_{i}$ preserves $\lambda$-presentable objects.

Proof. As in the proof of Theorem 3.1(3), we may assume that $\mathcal{M}_{i}, i \in I$, and $\mathcal{N}$ are full subcategories of the category of graphs Gra since each of them has a small dense subcategory. Assuming Vopěnka's principle, the inclusions $\mathcal{M}_{i} \rightarrow$ Gra, $i \in I$, and $\mathcal{N} \rightarrow$ Gra preserve $\lambda_{0}$-directed colimits for some $\lambda_{0}$, by [1, Theorem 6.9]. For $X \in \mathbf{G r a},|X|$ denotes the cardinality of the underlying set of the graph $X$. As before, given a regular cardinal $\alpha \geqslant \lambda_{0}$ and $X \in \mathcal{M}_{i}(X \in \mathcal{N}),|X|<\alpha$ implies that $X$ is $\alpha$-presentable in $\mathcal{M}_{i}$ (in $\mathcal{N}$ ). Moreover, as already explained in the proof of Corollary 3.3(1), the proof of [2, Theorem 1] shows how to define a closed and unbounded class of cardinals $\mathbf{C}_{1}$ with the property that for each regular cardinal $\lambda \in \mathbf{C}_{1}$, we have: (a) for any $i \in I, X \in \mathcal{M}_{i}$ is $\lambda$-presentable if and only if $|X|<\lambda$, and (b) $Y \in \mathcal{N}$ is $\lambda$-presentable if and only if $|Y|<\lambda$.

For any cardinal $\alpha$, let $\alpha^{*} \geqslant \alpha$ be the smallest cardinal such that for each $X \in \mathcal{M}_{i}$ with $|X|<\alpha$, then $\left|F_{i}(X)\right|<\alpha^{*}$ for every $i \in I$. Let $\mathbf{C}_{2}$ be the class of all cardinals $\alpha \geqslant \lambda_{0}$ such that $\alpha=\alpha^{*}$. The class $\mathbf{C}_{2}$ is closed and unbounded (similarly to the proof in [2, Theorem 1]). The intersection $\mathbf{C}_{3}=\mathbf{C}_{1} \cap \mathbf{C}_{2}$ is again closed and unbounded. Since the class of all regular cardinals is stationary under Vopěnka's principle, it follows that $\mathbf{C}_{3}$ contains a regular cardinal $\lambda$ (see $[\mathbf{1 2}, \mathbf{2}]$ ). This $\lambda$ has the required property. Since $\lambda_{0}$ can be chosen to be arbitrarily large, the same applies to $\lambda$, too.

Example 3.8. Let $\mathscr{P}: \mathcal{S}$ et $\rightarrow \mathcal{S}$ et be the covariant power set functor. For an uncountable regular cardinal $\lambda$, the functor $\mathscr{P}$ preserves $\lambda$-presentable objects in $\mathcal{S}$ et if and only if $\lambda$ is inaccessible. Applying Proposition 3.7 to this functor, we can then deduce the well known fact that Vopěnka's principle implies the existence of arbitrarily large inaccessible cardinals (see [1, Appendix]). In fact, a careful examination of the arguments shows that the existence of arbitrarily large inaccessible cardinals follows also from the (strictly weaker) assertion that the class of all regular cardinals is stationary. The fact that this assertion is a consequence of Vopěnka's principle is easily deduced from [12, Theorem 3].

\subsection{Weak equivalences and filtered colimits}

Our next goal is to find general conditions under which the class of weak equivalences in a model category $\mathcal{M}$ is closed under large enough filtered colimits. The strategy follows the proof of [17, Proposition 4.1]. We begin with the following lemma about filtered colimits of trivial fibrations.

Lemma 3.9. Let $(\mathcal{M}, \mathcal{C} o f, \mathcal{W}, \mathcal{F} i b)$ be a model category. Suppose that $(\mathcal{C} \circ f, \mathcal{W} \cap \mathcal{F} i b)$ is strictly cofibrantly generated. Then, assuming Vopěnka's principle, there is a regular cardinal $\lambda$ such that given a $\lambda$-filtered diagram $F: P \rightarrow \mathcal{M}_{c}$ whose values are trivial fibrations in $\mathcal{M}$ and $\operatorname{colim}_{P} F \in \mathcal{M}_{c}$, then $\operatorname{colim}_{P} F$ is again a trivial fibration.

Proof. Let $\mathcal{X}$ be a generating set for $(\mathcal{C} \circ f, \mathcal{W} \cap \mathcal{F} i b)$ whose domains are strictly $\kappa$ small and let $\mathcal{A}=\mathcal{A}(\mathcal{X}, \kappa)$ be the associated set of objects. By Theorem $3.1, \mathcal{A}$ is a small colimit-dense subcategory of $\mathcal{M}_{c}$. Let $\mathcal{K}$ be the full subcategory of $\mathcal{M}$ obtained by adding the domains and the codomains of the morphisms in $\mathcal{X}$ to $\mathcal{M}_{c}$. The union 
of the domains and codomains of $\mathcal{X}$ with $\mathcal{A}$ defines a small colimit-dense subcategory of $\mathcal{K}$. Using the same argument as in the proof of Theorem 3.1(3), it follows that every object of $\mathcal{K}$ is presentable in $\mathcal{K}$. Then there exists a regular cardinal $\lambda$ such that the domains and the codomains of the morphisms in $\mathcal{X}$ are $\lambda$-presentable in $\mathcal{K}$. Consequently, trivial fibrations between cofibrant objects are closed under $\lambda$-filtered colimits which exist in $\mathcal{K}$. In particular, they are closed under those $\lambda$-filtered colimits that come from colimits in $\mathcal{M}$.

Remark 3.10. Note that an easier and direct argument shows that if $(\mathcal{C} \circ f, \mathcal{W} \cap \mathcal{F}$ ib) is perfectly cofibrantly generated, then the conclusion of Lemma 3.9 holds for all trivial fibrations without the assumption of Vopěnka's principle.

Remark 3.11. There is an analogous statements for fibrations assuming instead that $(\mathcal{C} o f \cap \mathcal{W}, \mathcal{F} i b)$ is strictly cofibrantly generated. The proof is similar to the proof of Lemma 3.9.

Proposition 3.12. Let $(\mathcal{M}, \mathcal{C} o f, \mathcal{W}, \mathcal{F} i b)$ be a model category. Suppose that $(\mathcal{C} o f$, $\mathcal{W} \cap \mathcal{F} i b)$ is strictly cofibrantly generated. Then, assuming Vopěnka's principle, there is a regular cardinal $\lambda$ such that given a $\lambda$-filtered diagram $F: P \rightarrow \mathcal{M}_{c}$ whose values are weak equivalences and $\operatorname{colim}_{P} F \in \mathcal{M}_{c}$, then $\operatorname{colim}_{P} F$ is again a weak equivalence.

Proof. Let $\lambda$ be a regular cardinal that satisfies the property stated in Lemma 3.9. It suffices to prove the claim for $\lambda$-directed colimits. Following Remark 2.6, for every $\lambda$-directed category $\mathcal{D}$, there is a cofinal functor $\mathcal{D}_{0} \rightarrow \mathcal{D}$ from a $\lambda$-directed good poset $\mathcal{D}_{0}$, so it suffices to prove the claim for $\lambda$-directed good colimits.

Let $P$ be a $\lambda$-directed good poset. The category $\mathcal{M}^{P}$ of $P$-diagrams in $\mathcal{M}$ admits a model structure where the weak equivalences and the fibrations are defined pointwise (see [10, Theorem 5.1.3]). Furthermore, every cofibration in $\mathcal{M}^{P}$ is also a pointwise cofibration. Then the colimit-functor

$$
\operatorname{colim}_{P}: \mathcal{M}^{P} \rightarrow \mathcal{M}
$$

is a left Quillen functor.

Let $F, G: P \rightarrow \mathcal{M}_{c}$ be two pointwise cofibrant $P$-diagrams whose colimits are in $\mathcal{M}_{c}$ and $\phi: F \rightarrow G$ a natural transformation which is a pointwise weak equivalence. There is a factorization of $\phi$ in $\mathcal{M}^{P}$

$$
F \stackrel{\iota}{\longrightarrow} T \stackrel{\pi}{\longrightarrow} G
$$

such that $\iota$ is a trivial cofibration and $\pi$ is a trivial fibration. The diagram $T: P \rightarrow$ $\mathcal{M}$ is also pointwise cofibrant. Since $\operatorname{colim}_{P}$ is a left Quillen functor, the induced morphism

$$
\operatorname{colim}_{P}(F) \rightarrow \operatorname{colim}_{P}(T)
$$

is a trivial cofibration. Therefore $\operatorname{colim}_{P}(T)$ is also cofibrant. By Lemma 3.9, the induced morphism

$$
\operatorname{colim}_{P}(T) \rightarrow \operatorname{colim}_{P}(G)
$$

is a trivial fibration (between cofibrant objects). Hence $\operatorname{colim}_{P}(\phi)$ is a weak equivalence as required. 
Remark 3.13. Following Remark 3.10 , if $(\mathcal{C} \circ f, \mathcal{W} \cap \mathcal{F} i b)$ is perfectly cofibrantly generated, then the conclusion of Proposition 3.12 holds for all weak equivalences without the assumption of Vopěnka's principle.

Corollary 3.14. Let $(\mathcal{M}, \mathcal{C}$ of $, \mathcal{W}, \mathcal{F} i b)$ and $\lambda$ be as in Proposition 3.12. Let $F: P \rightarrow$ $\mathcal{M}_{c}$ be a $\lambda$-filtered diagram such that $\operatorname{colim}_{P} F$ is cofibrant. Then the canonical morphism

$$
c: \operatorname{hocolim}_{P} F \longrightarrow \operatorname{colim}_{P} F
$$

is a weak equivalence.

Proof. Similarly to the proof of Proposition 3.12, that is, by (homotopy) cofinality, we may assume that $P$ is a $\lambda$-directed good poset. Let $\widetilde{F}: P \rightarrow \mathcal{M}_{c}$ be a cofibrant replacement of $F$ in the model category $\mathcal{M}^{P}$ where weak equivalences and fibrations are defined pointwise, and let $\eta: \widetilde{F} \rightarrow F$ denote the weak equivalence in $\mathcal{M}^{P}$. Then it suffices to show that the canonical morphism

$$
\operatorname{colim}_{P}(\eta): \operatorname{colim}_{P} \widetilde{F} \rightarrow \operatorname{colim}_{P} F
$$

is a weak equivalence. Note that $\operatorname{colim}_{P} \widetilde{F}$ is cofibrant. Then the result follows from Proposition 3.12.

\section{Small presentations of model categories}

A small presentation of a model category $\mathcal{M}$ consists of a small category $\mathcal{C}$, a set of morphisms $S$ in the projective model category $\mathcal{S} \mathcal{S e t}{ }^{\mathcal{C}^{\mathrm{op}}}$, and a Quillen equivalence

$$
F: L_{S} \mathcal{S} \mathcal{S} e t^{\mathcal{C}^{\text {op }}} \rightleftarrows \mathcal{M}: G .
$$

Here $\mathcal{S}$ Set denotes the usual combinatorial model category of simplicial sets and $L_{S} \mathcal{S} \mathcal{S}$ et ${ }^{\mathcal{C}^{\text {op }}}$ the left Bousfield localization of the projective model category $\mathcal{S} \mathcal{S} \mathrm{et}^{\mathcal{C}^{\mathrm{op}}}$ at the set $S$. It is well known that $\mathcal{S} \mathcal{S}$ et ${ }^{\text {op }}$ is a (proper, simplicial) combinatorial model category. Moreover, when $S$ is a set of morphisms, the left Bousfield localization $L_{S} \mathcal{S} \mathcal{S}$ et $^{\mathcal{C}^{\text {op }}}$ exists and defines a new (left proper, simplicial) combinatorial model category. The notion of a small presentation for model categories was introduced and studied by Dugger $[\mathbf{4}, \mathbf{5}]$. It is the model-categorical analogue of the notion of a presentation of a locally presentable category as a small orthogonality class in a presheaf category.

In this section, we obtain results about the existence of small presentations for model categories - assuming Vopěnka's principle or not. As a consequence, we conclude that suitable model categories are Quillen equivalent to a (left proper, simplicial) combinatorial model category. The strategy for obtaining such small presentations follows Dugger [5] who showed that every combinatorial model category admits a small presentation.

\subsection{Dugger's method}

Let $\mathcal{C}$ be a small category and $\mathcal{S} \mathcal{S}$ et ${ }^{\mathcal{C}^{\mathrm{op}}}$ the projective model category of simplicial presheaves on $\mathcal{C}$ where the weak equivalences and the fibrations are defined pointwise. Given a cocomplete category $\mathcal{M}$, a left adjoint functor $F: \mathcal{S} \mathcal{S e t}^{\mathcal{C}^{\mathrm{op}}} \rightarrow \mathcal{M}$ is 
determined, uniquely up to canonical isomorphism, by its restriction

$$
f=F \circ y: \mathcal{C} \times \Delta \rightarrow \mathcal{M}
$$

where $y: \mathcal{C} \times \Delta \rightarrow \mathcal{S} \mathcal{S}$ et $^{\mathcal{C}^{\mathrm{op}}}=\mathcal{S}$ et $^{(\mathcal{C} \times \Delta)^{\mathrm{op}}}$ is the Yoneda embedding.

Let $\mathcal{M}$ be a model category and $f: \mathcal{C} \times \Delta \rightarrow \mathcal{M}$ a diagram. The associated left adjoint functor $F: \mathcal{S S}$ et $^{\mathcal{C}^{\mathrm{OP}}} \rightarrow \mathcal{M}$ is left Quillen if and only if for each $c \in \mathcal{C}$, the cosimplicial object $f(c,[\bullet]) \in c \mathcal{M}:=\mathcal{M}^{\Delta}$ is a cosimplicial resolution in $c \mathcal{M}$, i.e., it is Reedy cofibrant and homotopically constant, see [4, Proposition 3.4]. Such a cosimplicial resolution is determined up to a contractible space of choices by its restriction to degree 0 , i.e., the diagram $f(-,[0]): \mathcal{C} \rightarrow \mathcal{M}, c \mapsto f(c,[0])$.

These observations suggest the following general way of constructing Quillen adjunctions $\mathcal{S S}$ et $^{\mathcal{C}^{\mathrm{op}}} \rightleftarrows \mathcal{M}$ (see also [5, Section 6]). Let $C$ be a small full subcategory of $\mathcal{M}_{c}$ and let $\Delta C$ denote the small full subcategory of $c \mathcal{M}$ which consists of cosimplicial resolutions $A^{\bullet} \in c \mathcal{M}$ such that $A^{n} \in C$ for all $n$. Then the functor $f: \Delta C \times \Delta \rightarrow \mathcal{M}$, $\left(A^{\bullet},[n]\right) \mapsto A^{n}$, extends to a Quillen adjunction

$$
F_{C}: \mathcal{S} \mathcal{S e t}^{\Delta C^{\mathrm{op}}} \rightleftarrows \mathcal{M}: G_{C} .
$$

We will refer to this Quillen adjunction as the canonical Quillen adjunction which is generated by $C \subset \mathcal{M}_{c}$.

The first step towards finding a small presentation for $\mathcal{M}$ is to find a left Quillen functor $F: \mathcal{S S}$ et $^{\mathcal{C}^{\text {op }}} \rightarrow \mathcal{M}$ which defines a homotopy reflection in the following sense.

Definition 4.1. A Quillen adjunction $F: \mathcal{S} \mathcal{S e t}^{\mathcal{C}^{\text {op }}} \rightleftarrows \mathcal{M}: G$ is a homotopy reflection if for every fibrant object $X \in \mathcal{M}$ and cofibrant replacement $G(X)^{c} \stackrel{\sim}{\rightarrow} G(X)$, the induced morphism $F\left(G(X)^{c}\right) \rightarrow X$ is a weak equivalence. That is, the induced derived adjunction $\mathbb{L} F: \operatorname{Ho}\left(\mathcal{S} \mathcal{S}\right.$ et $\left.{ }^{\mathcal{C}^{\mathrm{op}}}\right) \rightleftarrows \mathrm{Ho}(\mathcal{M}): \mathbb{R} G$ defines a reflection.

Such a left Quillen functor $F$ is called homotopically surjective in [5]. Following [5], one can identify the derived counit transformation of a Quillen adjunction $F: \mathcal{S} \mathcal{S} \mathrm{et}^{\mathcal{C}^{\mathrm{op}}} \rightleftarrows \mathcal{M}: G$ as follows. Let $f=F \circ y: \mathcal{C} \times \Delta \rightarrow \mathcal{M}$ be the restriction along the Yoneda embedding. For each $X \in \mathcal{M}$, we have the canonical diagram

$$
\mathcal{C} \times \Delta \downarrow X \longrightarrow \mathcal{M},((c,[n]), u) \mapsto f(c,[n]) .
$$

Let $\operatorname{hocolim}(\mathcal{C} \times \Delta \downarrow X)$ denote its homotopy colimit. In [5, 4.2-4.4], the derived counit transformation of $(F, G)$ is identified with the canonical natural morphism

$$
q_{X}: \operatorname{hocolim}(\mathcal{C} \times \Delta \downarrow X) \rightarrow X .
$$

Hence a Quillen adjunction $F: \mathcal{S} \mathcal{S e t}^{\mathcal{C}^{\text {op }}} \rightleftarrows \mathcal{M}: G$ is a homotopy reflection if and only if the canonical morphism $q_{X}$ is a weak equivalence for all fibrant $X$.

Using this identification and combining several results from [5], we obtain the following criterion for a canonical Quillen adjunction $\left(F_{C}, G_{C}\right)$ to be a homotopy refection. We recall that the category $c \mathcal{M}$ has a simplicial enrichment and it is tensored and cotensored over simplicial sets. For every cosimplicial resolution $A^{\bullet} \in c \mathcal{M}$ and simplicial set $K$, the cosimplicial object $A^{\bullet} \otimes K$ is again a cosimplicial resolution (see [6, Proposition 4.8]).

Proposition 4.2. Let $\mathcal{M}$ be a model category, $C$ be a small full subcategory of $\mathcal{M}_{c}$ and $\left(F_{C}, G_{C}\right): \mathcal{S} \mathcal{S e t}^{\Delta C^{\mathrm{op}}} \rightleftarrows \mathcal{M}$ the associated canonical Quillen adjunction. Suppose that the following are satisfied: 
(a) there is a functorial Reedy cofibrant replacement for cosimplicial objects in $C$, that is, a functor $R: c C \rightarrow c C$ such that $R\left(X^{\bullet}\right)$ is Reedy cofibrant and a natural weak equivalence $\eta: R\left(X^{\bullet}\right) \rightarrow X^{\bullet}$. Moreover, we require that $R\left(X^{\bullet}\right)^{0}=X^{0}$ and $\eta$ is the identity in degree 0 .

(b) for each $A^{\bullet} \in \Delta C$, then the cosimplicial resolution $A^{\bullet} \otimes \Delta^{1}$ is again in $\Delta C$. Then $\left(F_{C}, G_{C}\right)$ is a homotopy reflection if and only if the canonical morphism

$$
\operatorname{hocolim}(C \downarrow X) \rightarrow X
$$

is a weak equivalence for each fibrant-cofibrant object $X$.

Proof. Let $\Delta C^{n} \subset \Delta C \times \Delta$ be the subcategory of objects $\left(A^{\bullet},[n]\right)$ which is full in $\Delta C$ and constant in $\Delta$. Clearly, this is isomorphic to $\Delta C$ for each $n \geqslant 0$. We will write $\Delta C$ for $\Delta C^{0}$. Note that there are canonical functors $\Delta C^{n} \rightarrow \mathcal{M},\left(A^{\bullet},[n]\right) \mapsto A^{n}$. For each $X \in \mathcal{M}$, there is a natural functor $\Delta C \downarrow X \rightarrow \Delta C^{n} \downarrow X$ which is given by $\left(A^{\bullet}, A^{0} \rightarrow X\right) \mapsto\left(A^{\bullet}, A^{n} \rightarrow A^{0} \rightarrow X\right)$. Using property (b), the proof of [5, Lemma 6.4 ] shows that the canonical induced morphism

$$
\operatorname{hocolim}(\Delta C \downarrow X) \longrightarrow \operatorname{hocolim}\left(\Delta C^{n} \downarrow X\right)
$$

is a weak equivalence. Applying [5, Proposition 4.6], it follows that there is a canonical weak equivalence

$$
\operatorname{hocolim}(\Delta C \downarrow X) \simeq \operatorname{hocolim}(\Delta C \times \Delta \downarrow X) .
$$

Therefore $\left(F_{C}, G_{C}\right)$ is a homotopy reflection if and only if the canonical morphism

$$
\operatorname{hocolim}(\Delta C \downarrow X) \longrightarrow X
$$

is a weak equivalence for each fibrant $X \in \mathcal{M}$. This follows from the identification of the derived counit morphism [5, Proposition 4.2, Corollary 4.4]. Furthermore, using property (a), the proof of [5, Lemma 6.3$]$ shows that the canonical morphism induced by $\Delta C \rightarrow C, A^{\bullet} \mapsto A^{0}$,

$$
\operatorname{hocolim}(\Delta C \downarrow X) \longrightarrow \operatorname{hocolim}(C \downarrow X)
$$

is a weak equivalence. The restriction to cofibrant objects does not affect the argument by $[\mathbf{5}$, Corollary 4.3].

Remark 4.3. It is useful to note that the assumptions (a) and (b) in Proposition 4.2 are used separately in the proof for two different arguments. In particular, if only (b) is satisfied, then $\left(F_{C}, G_{C}\right)$ is a homotopy reflection if and only if the canonical morphism

$$
\operatorname{hocolim}(\Delta C \downarrow X) \rightarrow X
$$

is a weak equivalence for each fibrant-cofibrant $X$. Moreover, in the proof of Theorem 4.5(2) below, a weaker version of (a) will be sufficient.

Thus, in order to find a canonical Quillen adjunction which is a homotopy reflection, the strategy will first be to find a small full subcategory $C$ of $\mathcal{M}$ which satisfies the assumptions of Proposition 4.2. When this is done and we obtain a homotopy reflection, what remains is to address the problem of turning a homotopy reflection into a Quillen equivalence. The following generalizes [5, Proposition 3.2]. 
Proposition 4.4. Let $(\mathcal{M}, \mathcal{C} o f, \mathcal{W}, \mathcal{F} i b)$ be a model category, $\mathcal{C}$ be a small category, and

$$
F: \mathcal{S} \text { et }^{\mathcal{C}^{\mathrm{op}}} \rightleftarrows \mathcal{M}: G
$$

a homotopy reflection. Then:

(1) Assuming Vopěnka's principle, there is a set of morphisms $S \subset \mathcal{S S} \mathrm{et}^{\mathcal{C}^{\mathrm{op}}}$ and an induced Quillen equivalence $L_{S} \mathcal{S} \mathcal{S e t}^{\mathcal{C}^{\mathrm{op}}} \rightleftarrows \mathcal{M}$.

(2) The same conclusion holds, without the assumption of Vopernka's principle, if in addition $(\mathcal{C} o f, \mathcal{W} \cap \mathcal{F}$ ib) is perfectly cofibrantly generated and $(\mathcal{C} \circ f \cap \mathcal{W}, \mathcal{F}$ ib) is semi-perfectly cofibrantly generated.

Proof. For (1), let $S$ be the class of canonical morphisms

$$
X^{c} \rightarrow G\left(F\left(X^{c}\right)^{f}\right),
$$

where $X$ is in $\mathcal{S} \mathcal{S}$ et ${ }^{\mathcal{C}^{\mathrm{op}}}$ and $(-)^{c}$ and $(-)^{f}$ denote choices of cofibrant and fibrant replacement functors. By [20, Theorem 2.3] (see also [3]), the left Bousfield localization of $\mathcal{S} \mathcal{S}$ et ${ }^{\mathcal{C}^{\text {op }}}$ at $S$ exists under the assumption of Vopěnka's principle. Moreover, there is a set $S^{\prime} \subset S$ such that we have $L_{S} \mathcal{S} \mathcal{S}$ et ${ }^{\mathcal{C}^{\text {op }}}=L_{S^{\prime}} \mathcal{S} \mathcal{S}$ et ${ }^{\mathcal{C}^{\text {op }}}$. Then it is easy to conclude that the induced Quillen adjunction $L_{S} \mathcal{S} \mathcal{S e t}^{\mathcal{C}^{\mathrm{op}}} \rightleftarrows \mathcal{M}$ is a Quillen equivalence.

For (2), note first that there is a regular cardinal $\lambda$ such that the following are satisfied:

(i) There is a cofibrant replacement functor $(-)^{c}: \mathcal{S} \mathcal{S}$ et ${ }^{\mathcal{C}^{\mathrm{op}}} \rightarrow \mathcal{S S} \mathrm{e}^{\mathrm{C}^{\mathrm{op}}}$ which preserves $\lambda$-filtered colimits.

(ii) There is a fibrant replacement functor $(-)^{f}: \mathcal{M} \rightarrow \mathcal{M}$ which preserves $\lambda$-filtered colimits.

(iii) $(F \circ y)(c,[n]) \in \mathcal{M}_{c}$ is $\lambda$-presentable in $\mathcal{M}$ for each $(c,[n]) \in \mathcal{C} \times \Delta$.

Indeed, conditions (i)-(ii) are satisfied by the factorizations given by the small object argument, and condition (iii) follows from Corollary 3.4(2). Let $S$ be the set of morphisms

$$
X^{c} \rightarrow G\left(F\left(X^{c}\right)^{f}\right),
$$

where $X$ is $\lambda$-presentable in $\mathcal{S} \mathcal{S}$ et ${ }^{\mathcal{C}^{\mathrm{op}}}$. The left Bousfield localization $L_{S} \mathcal{S} \mathcal{S}$ et ${ }^{\mathcal{C}^{\text {op }}}$ exists and there is an induced Quillen adjunction $\bar{F}: L_{S} \mathcal{S} \mathcal{S}$ et ${ }^{\mathcal{C}^{\mathrm{op}}} \rightleftarrows \mathcal{M}: \bar{G}-$ this is again a homotopy reflection. We claim that the composite

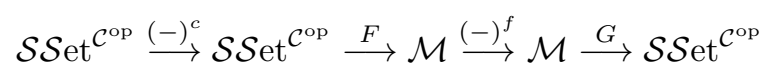

preserves $\lambda$-filtered colimits. Indeed, this follows from the assumptions since each of the functors preserves $\lambda$-filtered colimits. Therefore, if we write an arbitrary object $X \in \mathcal{S} \mathcal{S} \mathrm{et}^{\mathrm{C}^{\mathrm{op}}}$ as a $\lambda$-filtered colimit of $\lambda$-presentable objects, the canonical morphism $X^{c} \rightarrow G\left(F\left(X^{c}\right)^{f}\right)$ is a $\lambda$-filtered colimit of morphisms in $S$. Since weak equivalences in $\mathcal{S} \mathcal{S}$ et ${ }^{\mathrm{Cop}^{\mathrm{p}}}$ are closed under filtered colimits, it follows that $X^{c} \rightarrow G\left(F\left(X^{c}\right)^{f}\right)$ is an $S$-local equivalence for each $X$ and therefore $(\bar{F}, \bar{G})$ is a Quillen equivalence. 


\subsection{The main results}

The following theorem is our main result on the existence of small presentations for model categories. The first part assumes Vopěnka's principle and establishes a slightly weaker version of the claim in [16]. The second part is independent of Vopěnka's principle and generalizes the main result of [5].

Theorem 4.5. Let $(\mathcal{M}, \mathcal{C} o f, \mathcal{W}, \mathcal{F} i b)$ be a model category such that $(\mathcal{C} o f, \mathcal{W} \cap \mathcal{F} i b)$ is strictly cofibrantly generated.

(1) Assume that Vopěnka's principle holds. Then $\mathcal{M}$ admits a small presentation.

(2) Suppose that $(\mathcal{C} o f, \mathcal{W} \cap \mathcal{F} i b)$ is perfectly cofibrantly generated and $(\mathcal{C} \circ \cap \mathcal{W}$, $\mathcal{F} i b)$ is semi-perfectly cofibrantly generated. Then $\mathcal{M}$ admits a small presentation.

Proof. We first prove (1). By Corollary 3.4(3) and Propositions 3.7 and 3.12, it follows that there is a regular cardinal $\lambda$ such that

(i) $\mathcal{M}_{c}$ contains a small full subcategory $\mathcal{A}=\mathcal{A}(\mathcal{X}, \lambda)$ which consists of $\lambda$-presentable objects in $\mathcal{M}_{c}$ and each cofibrant object is a $\lambda$-filtered colimit in $\mathcal{M}$ of objects in $\mathcal{A}$.

(ii) there is a Reedy cofibrant replacement functor $R: c \mathcal{M}_{c} \rightarrow c \mathcal{M}_{c}$ which preserves cosimplicial objects which are pointwise $\lambda$-presentable in $\mathcal{M}_{c}$. Moreover, we may assume that $R\left(X^{\bullet}\right)^{0} \rightarrow X^{0}$ is the identity morphism for each $X^{\bullet} \in c \mathcal{M}_{c}$ (since $X^{0}$ is already cofibrant in $\left.\mathcal{M}\right)$.

(iii) the weak equivalences between cofibrant objects are closed under $\lambda$-filtered colimits in $\mathcal{M}$ which land in $\mathcal{M}_{c}$.

(i) follows from Corollary 3.4. Since $\mathcal{M}_{c}$ is preaccessible, and therefore has a small dense subcategory, it embeds fully in the category of graphs Gra. It follows that $c \mathcal{M}_{c}$ embeds fully in a locally presentable category. As a consequence, assuming Vopěnka's principle, $c \mathcal{M}_{c}$ is again preaccessible by $[\mathbf{1}$, Theorem 6.6] and [2, Theorem 1]. Let $R: c \mathcal{M}_{c} \rightarrow c \mathcal{M}_{c}$ be the standard Reedy cofibrant replacement functor which is defined inductively on the cosimplicial degree (leaving degree 0 unchanged). Then (ii) follows from Proposition 3.7 applied to the small collection of functors

$$
\left\{\mathrm{ev}_{n} R: c \mathcal{M}_{c} \stackrel{R}{\rightarrow} c \mathcal{M}_{c} \stackrel{\mathrm{ev}_{n}}{\rightarrow} \mathcal{M}_{c}\right\}_{n \geqslant 0} .
$$

Moreover, it is possible to find a common $\lambda$ satisfying simultaneously (i) and (ii) (cf. Remark 3.5). (iii) follows from Proposition 3.12.

Let $C$ be the small full subcategory of $\mathcal{M}_{c}$ consisting of $\lambda$-presentable objects. We recall that $\Delta C$ denotes the small full subcategory of $c \mathcal{M}$ which consists of cosimplicial resolutions $A^{\bullet} \in c \mathcal{M}$ such that $A^{n} \in C$ for each $n$. As explained earlier, the functor $\Delta C \times \Delta \rightarrow \mathcal{M},\left(A^{\bullet},[n]\right) \mapsto A^{n}$, extends to a left Quillen functor $F_{C}: \mathcal{S} \mathcal{S e t}^{\Delta C^{\text {op }}} \rightarrow \mathcal{M}$. We claim that this defines a homotopy reflection. Assumption (a) of Proposition 4.2 is satisfied by (ii) above. Assumption (b) of Proposition 4.2 is satisfied since $\left(A^{\bullet} \otimes \Delta^{1}\right)^{n}$ is the coend (in $\left.\mathcal{M}\right) A^{\bullet} \otimes_{\Delta}\left(\Delta^{1} \times \Delta^{n}\right) \in \mathcal{M}_{c}$, and therefore the presentability rank is preserved (this is also a coend in $\mathcal{M}_{c}$ ).

Therefore, by Proposition 4.2, it suffices to show that for each fibrant-cofibrant 
object $X \in \mathcal{M}$, the canonical morphism

$$
\operatorname{hocolim}(C \downarrow X) \longrightarrow X
$$

is a weak equivalence. We have canonical morphisms

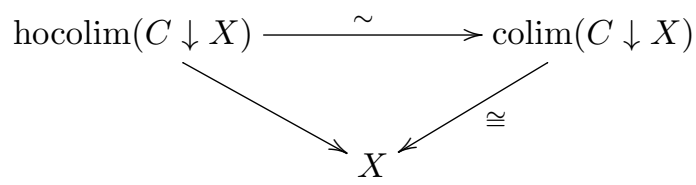

The object $X$ can be written as a $\lambda$-filtered colimit of $G: I \rightarrow \mathcal{M}$ with values in $\mathcal{A} \subseteq C$ - this is also a colimit in $\mathcal{M}_{c}$. It follows that the functor

$$
I \rightarrow C \downarrow X, \quad i \mapsto(G(i) \rightarrow X)
$$

is cofinal. Hence $C \downarrow X$ is also $\lambda$-filtered and the right morphism in the diagram above is an isomorphism by cofinality. Moreover, the horizontal morphism is a weak equivalence by (iii) above and Corollary 3.14. It follows that the left morphism is a weak equivalence for each fibrant-cofibrant $X$, and therefore $F$ defines a homotopy reflection as claimed. The result then follows by applying Proposition 4.4(1).

For (2), the argument is analogous - but a little different. Again, there is a regular cardinal $\lambda$ such that

(i) $\mathcal{M}_{c}$ contains a small full subcategory $\mathcal{A}=\mathcal{A}(\mathcal{X}, \lambda)$ which consists of $\lambda$-presentable objects in $\mathcal{M}$ and each cofibrant object is a $\lambda$-filtered colimit in $\mathcal{M}$ of objects in $\mathcal{A}$.

(ii) there is a Reedy cofibrant replacement functor $R: c \mathcal{M}_{c} \rightarrow c \mathcal{M}_{c}$ such that: (a) $R$ sends each constant cosimplicial object at a $\lambda$-presentable cofibrant object to a cosimplicial object which is pointwise $\lambda$-presentable in $\mathcal{M}$, and (b) $R$ preserves Reedy cofibrant objects which are pointwise $\lambda$-presentable in $\mathcal{M}$.

(iii) the weak equivalences are closed under $\lambda$-filtered colimits in $\mathcal{M}$.

(i) and (iii) are guaranteed by Corollary 3.4(2) and Remark 3.13 respectively. To see (ii), we need to consider the model category $c \mathcal{M}$ with the Reedy model structure. The weak factorization system $\left(\mathcal{C}_{o} f_{c \mathcal{M}}, \mathcal{W}_{c \mathcal{M}} \cap \mathcal{F} i b_{c \mathcal{M}}\right)$ of this Reedy model category $c \mathcal{M}$ is again perfectly cofibrantly generated (see [9, Proposition 15.6.24]). Even though (ii) does not follow directly from Corollary 3.3(2), the same idea of proof can be applied in this case. We claim that the functorial Reedy cofibrant replacement functor $\widetilde{R}: c \mathcal{M} \rightarrow c \mathcal{M}$ that arises from the small object argument restricts to a functor $R: c \mathcal{M}_{c} \rightarrow c \mathcal{M}_{c}$ with the desired properties. Indeed the properties are satisfied by applying Proposition 3.6 to the collection of functors

$$
\left\{\mathrm{ev}_{n} \widetilde{R}: c \mathcal{M} \stackrel{\widetilde{R}}{\longrightarrow} c \mathcal{M} \stackrel{\mathrm{ev}_{n}}{\longrightarrow} \mathcal{M}\right\}_{n \geqslant 0}
$$

and using that both $\mathcal{M}_{c}$, as a subcategory of constant cosimplicial objects in $c \mathcal{M}$, and $(c \mathcal{M})_{c} \subseteq c \mathcal{M}$ are preaccessibe in $c \mathcal{M}$ by Corollary 3.4.

Let $C$ be the small full subcategory of $\mathcal{M}_{c}$ which consists of the cofibrant objects which are $\lambda$-presentable objects in $\mathcal{M}$. Every object $X \in C$ is a $\lambda$-filtered colimit of objects in $\mathcal{A}$ and therefore also a split subobject of an object in $\mathcal{A}$. We consider the canonical Quillen adjunction $F_{C}: \mathcal{S} \mathcal{S}$ et ${ }^{\Delta C^{\text {op }}} \rightleftarrows \mathcal{M}: G_{C}$. Using (i), (iii) and similar 
arguments as before, it follows that the category $C \downarrow X$ is $\lambda$-filtered for every fibrantcofibrant $X$ and the canonical morphisms

$$
\operatorname{hocolim}(C \downarrow X) \stackrel{\sim}{\rightarrow} \operatorname{colimit}(C \downarrow X) \stackrel{\cong}{\longrightarrow} X
$$

are weak equivalences. Assumption (b) of Proposition 4.2 is satisfied, but we don't know about Assumption (a). According to the proof of Proposition 4.2 and Remark 4.3, the canonical Quillen adjunction $\left(F_{C}, G_{C}\right)$ is a homotopy reflection if the canonical morphism

$$
\operatorname{hocolim}(\Delta C \downarrow X) \longrightarrow X
$$

is a weak equivalence for every fibrant-cofibrant $X$. There is a functor $\sigma: \Delta C \downarrow X \rightarrow$ $C \downarrow X$ given by $\left(E^{\bullet}, E^{0} \rightarrow X\right) \mapsto\left(E^{0}, E^{0} \rightarrow X\right)$. The result will follow if we show that this functor induces a weak equivalence

$$
\operatorname{hocolim}(\Delta C \downarrow X) \simeq \operatorname{hocolim}(C \downarrow X) .
$$

The proof of [5, Lemma 6.3] applies under the present slightly weaker assumptions. The functor $R$ from (ii) above defines a functor

$$
\begin{aligned}
\rho: C \downarrow X & \longrightarrow \Delta C \downarrow X \\
(E, E \rightarrow X) & \mapsto\left(R(c E), R(c E)^{0} \rightarrow E \rightarrow X\right) .
\end{aligned}
$$

The composite functor $\sigma \circ \rho$ is connected to the identity functor by a natural transformation which is defined by the natural weak equivalence $R(c E)^{0} \rightarrow E$. We claim that the composite $\rho \circ \sigma$ is also connected to the identity via a zigzag of natural transformations. Let $H: \Delta C \downarrow X \rightarrow \Delta C \downarrow X$ be the functor given by

$$
\left(E^{\bullet}, E^{0} \rightarrow X\right) \mapsto\left(R\left(E^{\bullet}\right), R\left(E^{\bullet}\right)^{0} \rightarrow E^{0} \rightarrow X\right) .
$$

This is well defined by assumption (ii). Moreover, there are natural transformations

$$
\begin{aligned}
& H \rightarrow \mathrm{Id}, \\
& H \rightarrow \rho \circ \sigma .
\end{aligned}
$$

The first one is induced by the natural weak equivalence $R\left(E^{\bullet}\right) \rightarrow E^{\bullet}$. The second natural transformation is defined by the natural weak equivalence $R\left(E^{\bullet}\right) \rightarrow R\left(c E^{0}\right)$. By [5, Proposition A.4], it follows that $\sigma$ induces a weak equivalence

$$
\operatorname{hocolim}(\Delta C \downarrow X) \simeq \operatorname{hocolim}(C \downarrow X)(\simeq X) .
$$

As a consequence, the Quillen adjunction $\left(F_{C}, G_{C}\right)$ is a homotopy reflection. The result then follows from Proposition 4.4(2).

Specializing to cofibrantly generated model categories, we obtain the following as immediate corollary.

Corollary 4.6. Let $(\mathcal{M}, \mathcal{C} o f, \mathcal{W}, \mathcal{F} i b)$ be a cofibrantly generated model category.

(1) Suppose that $(\mathcal{C} \circ f, \mathcal{W} \cap \mathcal{F} i b)$ is strictly cofibrantly generated. Assuming Vopernka's principle, $\mathcal{M}$ admits a small presentation.

(2) Suppose that $(\mathcal{C} o f, \mathcal{W} \cap \mathcal{F} i b)$ is $\aleph_{0}$-cofibrantly generated. Assuming Vopěnka's principle, $\mathcal{M}$ admits a small presentation. 
(3) Suppose that $(\mathcal{C} o f, \mathcal{W} \cap \mathcal{F} i b)$ is perfectly cofibrantly generated and $(\mathcal{C} \circ \cap \cap \mathcal{W}$, $\mathcal{F} i b)$ is semi-perfectly cofibrantly generated. Then $\mathcal{M}$ admits a small presentation.

In particular, in each of the cases (1)-(3), the model category $\mathcal{M}$ is Quillen equivalent to a combinatorial model category.

Remark 4.7. We do not know if the conclusion in Corollary 4.6 is true for all cofibrantly generated model categories.

\subsection{An example}

Theorem 4.5 is false for model categories whose weak factorization systems are only weakly cofibrantly generated. This was shown in [19] assuming the negation of Vopěnka's principle, but the claim holds more generally under the standard settheoretical assumptions.

Let $\mathcal{K}$ be the category of compact Hausdorff spaces with the trivial model structure where the weak equivalences are the isomorphisms. By Example 2.10, this model structure has weakly cofibrantly generated weak factorization systems. It follows from the results in Subsections 4.1 and 4.2 that every combinatorial model category has a small homotopically dense subcategory. Since $\mathcal{K}$ does not have a small (homotopically) dense subcategory, it is easy to conclude that it is not Quillen equivalent to a combinatorial model category.

Moreover, we claim that $\mathcal{K}$ is not equivalent to the homotopy category of a combinatorial model category. To see this, we begin with the following property of these homotopy categories which will serve as a basic criterion (cf. [18, Proposition 6.10]).

Proposition 4.8. Let $\mathcal{M}$ be a combinatorial model category and $\gamma: \mathcal{M} \rightarrow \operatorname{Ho}(\mathcal{M})$ the localization functor. Then there is a regular cardinal $\lambda$ such that for each $\lambda$-presentable object $X \in \mathcal{M}$, the object $\gamma(X)$ is $\lambda$-presentable with respect to coproducts in $\operatorname{Ho}(\mathcal{M})$, i.e., any morphism $\gamma(X) \rightarrow \coprod_{i \in I} Y_{i}$ in $\operatorname{Ho}(\mathcal{M})$ factors through a subobject $\coprod_{i \in J} Y_{i}$ with $|J|<\lambda$.

Proof. Let $\lambda$ be a regular cardinal such that the fibrant and cofibrant replacement functors in $\mathcal{M}$ given by the small object argument preserve $\lambda$-filtered colimits and $\lambda$-presentable objects. We denote these functors by $(-)^{c},(-)^{f}$, and $(-)^{c f}:=(-)^{f} \circ$ $(-)^{c}$. The existence of such $\lambda$ follows from [1, Theorem 2.19].

Let $X$ be a $\lambda$-presentable object in $\mathcal{M}$ and $f: \gamma(X) \rightarrow Y=\coprod_{i \in I}^{\mathrm{Ho}(\mathcal{M})} Y_{i}$ a morphism in $\operatorname{Ho}(\mathcal{M})$. Coproducts of cofibrant objects define coproducts in the homotopy category, hence $Y$ is canonically isomorphic to $\gamma\left(\coprod_{i \in I}^{\mathcal{M}} Y_{i}^{c}\right)$. The associated morphism $f^{\prime}: \gamma(X) \rightarrow \gamma\left(\coprod_{i \in I}^{\mathcal{M}} Y_{i}^{c}\right)$ can be represented, up to canonical isomorphism in $\operatorname{Ho}(\mathcal{M})$, by a morphism $\tilde{f}: X^{c f} \rightarrow\left(\coprod_{i \in I}^{\mathcal{M}} Y_{i}^{c}\right)^{f}$. Since $(-)^{f}$ preserves $\lambda$-filtered colimits, the target of $\tilde{f}$ can be written as a $\lambda$-filtered colimit of $\left(\coprod_{j \in J}^{\mathcal{M}} Y_{j}^{c}\right)^{f}$ for all $J \subseteq I$ with $|J|<\lambda$. Then since $X^{c f}$ is again $\lambda$-presentable in $\mathcal{M}$, the morphism $\tilde{f}$ factors through $\left(\coprod_{j \in J}^{\mathcal{M}} Y_{j}^{c}\right)^{f}$ for some $J \subseteq I$ with $|J|<\lambda$. This factorization induces the required factorization in the homotopy category.

Suppose that $\operatorname{Ho}(\mathcal{K}) \cong \mathcal{K}$ is equivalent to $\operatorname{Ho}(\mathcal{M})$ where $\mathcal{M}$ is a combinatorial model category. Let $\gamma: \mathcal{M} \rightarrow \operatorname{Ho}(\mathcal{M})$ be the localization functor to the homotopy 
category $\operatorname{Ho}(\mathcal{M})$. Since the property in Proposition 4.8 is preserved by equivalences of categories, $\mathcal{K}$ must contain many objects which are $\lambda$-presentable with respect to coproducts. But $\emptyset$ is actually the only such object in $\mathcal{K}$, hence the contradiction. Indeed, there are maps $*=\mathbb{F}(*) \rightarrow \mathbb{F}(I)$ which do not factor through any $\mathbb{F}(J)$ with $|J|<\lambda$. To see this, first recall that the inclusion $\mathbb{F}(J) \rightarrow \mathbb{F}(I)$ sends an ultrafilter $\mathcal{U}$ on $J$ to the ultrafilter consisting of all subsets $X \subseteq I$ whose intersection $X \cap J$ belongs to $\mathcal{U}$. Then take an ultrafilter on $I$ containing the filter of subsets whose complement has cardinality $<\lambda$. This defines a point in $\mathbb{F}(I)$ which does not factor through any $\mathbb{F}(J)$ with $|J|<\lambda$.

\section{Erratum}

Let $\mathcal{M}$ be a cocomplete category and $S$ a set of objects. We denote $S$ also the full subcategory of $\mathcal{M}$ spanned by $S$. An object $X \in \mathcal{M}$ is called $S$-generated (see [16]) if the canonical morphism from the canonical diagram of $X$ with respect to $S$,

$$
\kappa_{S}(X):=\operatorname{colim}(S \downarrow X \rightarrow \mathcal{M}) \rightarrow X,
$$

is an isomorphism. Let $\mathcal{M}_{S}$ denote the full subcategory of $\mathcal{M}$ spanned by the $S$ generated objects. It was claimed in [16, Proposition 3.1(i)] that the functor $\kappa_{S}: \mathcal{M} \rightarrow$ $\mathcal{M}$ defines a right adjoint to the inclusion functor $\mathcal{M}_{S} \rightarrow \mathcal{M}$, but this claim is false in this generality. Indeed, the values of $\kappa_{S}$ are not $S$-generated objects in general.

The main purpose of considering the full subcategory $\mathcal{M}_{S}$ for the proof of the main result in [16, Theorem 1.1] was in order to obtain a coreflective subcategory of $\mathcal{M}$ which contains $S$ and has a small dense subcategory, cf. [16, Proposition 3.1(iii)]. But the existence of such a coreflective category cannot be guaranteed in general, therefore also the proof of [16, Theorem 1.1] does not apply without additional assumptions.

Here is a general construction of counterexamples to these claims.

Example 5.1. Let $\mathcal{M}$ be a cocomplete category and $S$ a set of objects such that the colimit-closure of $S$ in $\mathcal{M}$ is $\mathcal{M}$, obtained possibly after using iterated colimits, but $\mathcal{M}$ does not have a small dense subcategory. Such examples exist, e.g., the category of compact Hausdorff spaces is the colimit-closure of the one-point space, but it does not have a small dense subcategory. Then $\mathcal{M}_{S}$ cannot be $\mathcal{M}$, because $\mathcal{M}_{S}$ has a small dense subcategory, but then it cannot be closed under colimits in $\mathcal{M}$. Therefore, $\mathcal{M}_{S}$ is not coreflective in $\mathcal{M}$.

Under the special assumption that such a coreflection exists, the proof of $[\mathbf{1 6}$, Theorem 1.1] applies to give the following corrected statement. We say that a model category $\mathcal{M}$ is setwise cofibrantly generated if both of its weak factorization systems are setwise cofibrantly generated.

Theorem 5.2. Let $\mathcal{M}$ be a setwise cofibrantly generated model category and let $S$ be the set of domains and codomains of the morphisms in the sets of generators for fibrations and trivial fibrations. Suppose that the inclusion functor $\mathcal{M}_{S} \rightarrow \mathcal{M}$ admits a right adjoint. Assuming Vopěnka's principle, then:

(1) $\mathcal{M}_{S}$ is locally presentable.

(2) $\mathcal{M}_{S}$ carries a cofibrantly generated model structure, inherited from $\mathcal{M}$, and the inclusion functor $\mathcal{M}_{S} \rightarrow \mathcal{M}$ is a Quillen equivalence. 
Proof. The proof follows the proof of [16, Theorem 1.1]. $\mathcal{M}_{S}$ is a cocomplete category with a small dense subcategory spanned by $S$. Assuming Vopěnka's principle, $\mathcal{M}_{S}$ is locally presentable by $[\mathbf{1}$, Theorem 6.14$]$.

The setwise cofibrantly generated model structure on $\mathcal{M}$ restricts to a cofibrantly generated model structure on $\mathcal{M}_{S}$ where the factorizations are given by the small object argument performed in $\mathcal{M}_{S}$.

Let $G: \mathcal{M} \rightarrow \mathcal{M}_{S}$ denote the right adjoint. It is easy to see that the counit morphism $G(X) \rightarrow X$ is a trivial fibration for all $X \in \mathcal{M}$. In particular, $G$ preserves the weak equivalences. Then it follows easily that the inclusion $\mathcal{M}_{S} \rightarrow \mathcal{M}$ is a left Quillen equivalence.

Remark 5.3. If the generators of the weak factorization systems in $\mathcal{M}$ permit the small object argument in $\mathcal{M}_{S}$, then (2) holds without the assumption of Vopěnka's principle.

The special assumption of Theorem 5.2 is satisfied for topological fiber-small categories, see [7]. Moreover, in this case, Vopěnka's principle is not needed. It would be interesting to identify also other classes of model categories for which the special assumption of Theorem 5.2 is satisfied.

Corollary 5.4. Let $\mathcal{M}$ be a setwise cofibrantly generated model category whose underlying category is a topological fiber-small category. Then $\mathcal{M}$ is Quillen equivalent to a combinatorial model category.

Proof. Let $\mathcal{I}$ be a generator for trivial fibrations and $\mathcal{J}$ a generator for fibrations. Let $S$ be the set of objects that appear as domains or codomains of the morphisms in $\mathcal{I} \cup \mathcal{J}$. The full subcategory $\mathcal{M}_{S}$ of $\mathcal{M}$ consisting of $S$-generated objects is locally presentable and coreflective in $\mathcal{M}$ [7, Proposition 3.5 and Theorem 3.6]. Similarly to Theorem 5.2, $\mathcal{M}_{S}$ inherits a cofibrantly generated model structure from $\mathcal{M}$ and the adjunction $\mathcal{M}_{S} \rightleftarrows \mathcal{M}$ is a Quillen equivalence.

\section{References}

[1] J. Adámek and J. Rosický, Locally Presentable and Accessible Categories. London Mathematical Society Lecture Note Series, 189. Cambridge University Press, Cambridge, 1994.

[2] J. Adámek and J. Rosický, On preaccessible categories, J. Pure Appl. Algebra 105 (1995), no. 3, 225-232.

[3] C. Casacuberta and B. Chorny, The orthogonal subcategory problem in homotopy theory, Contemp. Math. 399 (2006), 41-53.

[4] D. Dugger, Universal homotopy theories, Adv. Math. 164 (2001), no. 1, 144176.

[5] D. Dugger, Combinatorial model categories have presentations, Adv. Math. 164 (2001), no. 1, 177-201.

[6] D. Dugger, Replacing model categories with simplicial ones, Trans. Amer. Math. Soc. 353 (2001), no. 12, 5003-5027. 
[7] L. Fajstrup and J. Rosický, A convenient category for directed homotopy, Theory Appl. Categ. 21 (2008), no. 1, 7-20.

[8] R. Fraïssé, Theory of Relations. Studies in Logic and the Foundations of Mathematics, 145. North-Holland Publishing Co., Amsterdam, 2000.

[9] P.S. Hirschhorn, Model Categories and Their Localizations. Mathematical Surveys and Monographs, 99. American Mathematical Society, Providence, RI, 2003.

[10] M. Hovey, Model Categories. Mathematical Surveys and Monographs, 63. American Mathematical Society, Providence, RI, 1999.

[11] J. Lurie, Higher Topos Theory. Annals of Mathematics Studies, 170. Princeton University Press, Princeton, NJ, 2009.

[12] M. Magidor, On the role of supercompact and extendible cardinals in logic, Israel J. Math. 10 (1971), 147-157.

[13] M. Makkai and R. Paré, Accessible Categories: The Foundations of Categorical Model Theory. Contemporary Mathematics, 104. American Mathematical Society, Providence, RI, 1989.

[14] M. Makkai, J. Rosický and L. Vokřínek, On a fat small object argument, Adv. Math. 254 (2014), 49-68.

[15] D.G. Quillen, Homotopical Algebra. Lecture Notes in Mathematics, 43. Springer-Verlag, Berlin, New York, 1967.

[16] G. Raptis, On the cofibrant generation of model categories, J. Homotopy Relat. Struct. 4 (2009), no. 1, 245-253.

[17] G. Raptis and J. Rosický, The accessibility rank of weak equivalences, Theory Appl. Categ. 30 (2015), no. 19, 687-703.

[18] J. Rosický, Generalized Brown representability in homotopy categories, Theory Appl. Categ. 14 (2005), no. 19, 451-479.

[19] J. Rosický, Are all cofibrantly generated model categories combinatorial? Cah. Topol. Géom. Différ. Catég. 50 (2009), no. 3, 233-238.

[20] J. Rosický and W. Tholen, Left-determined model categories and universal homotopy theories, Trans. Amer. Math. Soc. 355 (2003), no. 9, 3611-3623.

G. Raptis georgios.raptis@ur.de

Fakultät für Mathematik, Universität Regensburg, 93040 Regensburg, Germany

J. Rosický rosicky@math.muni.cz

Department of Mathematics and Statistics, Faculty of Sciences, Masaryk University, Kotlářská 2, 61137 Brno, Czech Republic 